\title{
Probabilistic speed-density relationship for pedestrian traffic
}

\author{
Marija Nikolića, $^{a, *}$ Michel Bierlaire ${ }^{\mathrm{a}}$, Bilal Farooq ${ }^{\mathrm{b}}$, Matthieu de Lapparent $^{\mathrm{a}}$ \\ ${ }^{a}$ Transport and Mobility Laboratory, School of Architecture, Civil and Environmental Engineering, \\ École Polytechnique Fédérale de Lausanne, Station 18, CH-1015, Lausanne, Switzerland, \\ \{marija.nikolic, michel.bierlaire, matthieu.delapparent\}@epfl.ch \\ ${ }^{b}$ Département des génies civil, géologique et des mines, Polytechnique Montréal, $2500 \mathrm{Ch}$. \\ Polytechnique Montréal, H3T 1J4, Montréal, Canada, bilal.farooq@polymtl.ca
}

\begin{abstract}
We propose a probabilistic modeling approach to represent the speed-density relationship of pedestrian traffic. The approach is data-driven, and it is motivated by the presence of high scatter in the raw data that we have analyzed. We show the validity of the proposed approach, and its superiority compared to deterministic approaches from the literature using a dataset collected from a real scene and another from a controlled experiment.
\end{abstract}

Keywords: speed-density relationship, probabilistic model, individual trajectories, Voronoi tessellations, statistical validation

\section{Introduction}

Understanding, reproducing and forecasting phenomena that characterize pedestrian traffic is necessary in order to provide services related to pedestrian safety and convenience. This becomes of utmost importance in areas of high congestion, which is a growing problem of many public spaces (transportation hubs, shopping malls, large sports and cultural events, etc.). Congestion in pedestrian facilities represents a phenomenon with a negative impact on pedestrian dynamics. It prevents pedestrians from achieving efficient movements and may lead to an increase in travel time, delays and potential collisions among pedestrians.

Data collection for pedestrian flow and behavior analysis used to be particularly cumbersome. Typically, manual counting methods (on-site or on videos) and surveys distributed to randomly selected individuals were the main sources of data. Nowadays, automatic pedestrian detection and tracking methods have evolved tremendously, allowing for more comprehensive analyses (Bauer et al. 2009).

Using a direct analogy with vehicular traffic, the main stream of the literature characterizes pedestrian traffic with three fundamental quantities, that is density $(k)$, speed $(v)$ and flow $(q)$, as well as deterministic relationships among them. Density (in ped $/ \mathrm{m}^{2}$ ) is

* Corresponding author

Preprint submitted to Transportation Research Part B: Methodological

April 1, 2016 
the number of pedestrians present in an area at a given moment in time; speed (in $\mathrm{m} / \mathrm{s}$ ) may be averaged over space or over time; and flow (in ped/ms) refers to the number of pedestrians passing a cross section of an area per unit of time (Daamen 2004). Assuming that the traffic system is at equilibrium (stationary and homogenous), the relationships between density and flow, density and speed, and flow and speed is referred to as the fundamental diagram (Weidmann, 1993; Daamen, 2004).

In this paper, we propose a methodology to represent the speed-density relationship of pedestrian traffic in a probabilistic way. This is motivated by the analysis of data collected from the train station in Lausanne, Switzerland, as well as data collected from a controlled experiment by the Technical University of Delft (Daamen and Hoogendoorn. 2003). The empirical analysis of these pieces of data rules out the use of a unique deterministic fundamental diagram, due to a high scatter in the data. A possible approach to capture this complex phenomenon consists in modeling explicitly the exact underlying walking process and the explicit interactions at the disaggregate level. It would allow to test various hypotheses, at the expense of the collection of a great deal of disaggregate data. We propose in this paper an alternative approach, based on an aggregate representation of the pedestrian traffic. The scatter is explicitly represented by relaxing the homogeneity assumption of the equilibrium speed-density relationship.

The structure of the paper is as follows. A review of the literature is provided in Section 2 In Section 3, we first define the variables involved in the model. We then derive the macroscopic relationships between these indicators, starting from first principles. Based on this theoretical model, we introduce a probabilistic speed-density relationship in Section 4 Section 5 presents the two case studies from Lausanne and Delft mentioned above. It reports the empirical analysis of the two case studies and emphasizes the limitations of the state of the art approaches on these concrete examples. Section 6 and Section 7 illustrate the proposed model on the two case studies. Parameter estimation, model validation and comparisons with the existing models are discussed in details. Section 8 summarizes the outcomes of the proposed methodology and suggests future research directions.

\section{Literature review}

This section is organized into two parts. The first focuses on models from the vehicular traffic theory, that are relevant for pedestrian as well. The seconds deals with models for the pedestrian traffic.

\subsection{Vehicular traffic}

The fundamental diagram that corresponds to stationary homogenous traffic was first introduced empirically by Greenshields et al. (1935). This study established the relationship between spacing (the inverse of density) and speed in a form of a simple linear equation. Since then there have been many empirical studies that were aimed at improving this relationship. A comprehensive review of the models proposed in this field is given in Wang et al. (2009). Some of the established deterministic empirical 
relationships are listed in Table 1 where $v_{f}$ is the free-flow speed, $v_{0}$ is the average travel speed in stop-and-go conditions, $k_{j}$ is the jam density, $k_{c}$ is the critical density, and $\lambda, \theta$, $\theta_{1}$ and $\theta_{2}$ are parameters.

\begin{tabular}{|l|l|l|}
\hline Source & Specification & Parameters \\
\hline Greenshields et al. $(1935)$ & $v(k)=v_{f}\left(1-\frac{k}{k_{j}}\right)$ & $v_{f}, k_{j}$ \\
\hline \hline Underwood $(1961)$ & $v(k)=v_{f} \exp \left(-\frac{k}{k_{c}}\right)$ & $v_{f}, k_{c}$ \\
\hline Newell $(1961)$ & $v(k)=v_{f}\left(1-\exp \left(-\frac{\lambda}{v_{f}}\left(\frac{1}{k}-\frac{1}{k_{j}}\right)\right)\right)$ & $v_{f}, k_{j}, \lambda$ \\
\hline \hline Drake et al. $(1967)$ & $v(k)=v_{f} \exp \left(-\theta k^{2}\right)$ & $v_{f}, \theta$ \\
\hline Wang et al. $(2009)$ & $v(k)=v_{0}+\frac{v_{f}-v_{0}}{\left(1+\exp \left(\frac{k-k_{c}}{\theta_{1}}\right)\right)^{\theta_{2}}}$ & $v_{0}, v_{f}, k_{c}, \theta_{1}, \theta_{2}$ \\
\hline Units: $k[v e h / k m], v[k m / h]$ & \\
\hline
\end{tabular}

Table 1: Deterministic fundamental relationships - vehicular traffic

Another stream of the literature establishes the fundamental relations by analyzing the behavior of microscopic car-following models under the equilibrium conditions at the aggregate level. Different car-following models result in different specifications of fundamental relations (Jabari et al., 2014)..

In several recent studies, it has been recognized that plots of speed-density or flowdensity data are usually widely scattered. Wang et al.(2009) addressed this phenomenon through the probabilistic extension of the existing macroscopic relations. Treiber and Helbing (2003) explained the observed scattering by non-stationary dynamical aspects of the traffic system. In Kim and Zhang (2008) the impact of driver heterogeneity on the scatter in the fundamental relations is demonstrated. Jabari et al. (2014) also addressed this scatter through the modeling of macroscopic flow characteristics dependent on drivers' characteristics. The modeling approaches that take this observation into account are shown to explain the data better at the macroscopic level (Jabari et al. 2014).

\subsection{Pedestrian traffic}

In the context of pedestrian traffic, fundamental relations are usually established by fitting deterministic curves to empirical data. Both linear and nonlinear speed-density models have been proposed, as reported in Table 2 where $v_{f}$ is the free flow speed, $k_{j}$ the jam density, and $\theta$ and $\gamma$ are parameters. The linearity of the speed-density relationship has long been questioned for both vehicular and pedestrian flows (Daamen, 2004). An alternative specification has been proposed by Tregenza (1976) where speed decreases exponentially with the increase in density, whereas Weidmann (1993) proposed the so-called Kladek-formula, with a double S-form. The exponential specifications of the relationship appeared to be better for describing the behavior of pedestrian walking speed (Cheah and Smith, 1994). In comparison to fundamental relationships from vehicular traffic, the relationship proposed by Weidmann (1993) corresponds to the model proposed by Newell (1961), while the relationship proposed by Tregenza (1976) can be regarded as the generalization of the model proposed by Underwood (1961). Rastogi et al. (2013) have 
shown that the speed-density relationship of pedestrian flow on sidewalks also follows the model presented in Underwood (1961).

The proposed relationships clearly differ in terms of functional form, but also in terms of the values of their parameters and supports. For instance, jam density (the maximum density achieved under congestion) goes from $3.8 \mathrm{ped} / \mathrm{m}^{2}$ to $10 \mathrm{ped} / \mathrm{m}^{2}$, the reported critical density (the maximum density achievable under free flow) ranges from $1.7 \mathrm{ped} / \mathrm{m}^{2}$ to $7 \mathrm{ped} / \mathrm{m}^{2}$ (Seyfried et al. 2010) and the mean of the free-flow speed estimated in different studies is $1.34 \mathrm{~m} / \mathrm{s}$ while its standard deviation is $0.37 \mathrm{~m} / \mathrm{s}$ (Daamen 2004). The researchers have suggested several explanations for these deviations: the cultural differences, the differences between pedestrian facilities and the effects of the environment, flow composition, measurement methods, etc. (Seyfried et al. 2010).

\begin{tabular}{|l|l|l|}
\hline Source & Specification & Parameters \\
\hline Older $(\overline{1968})$ & & \\
\hline Navin and Wheeler $(1969)$ & & $v_{f}, \theta$ \\
\hline Fruin (1971) & $v(k)=v_{f}-\theta k$ \\
\hline Tanaboriboon et al. $(1986)$ & & $v_{f}, \theta$ \\
\hline Lam et al. $(1995)$ & $v(k)=v_{f}-v_{f} \theta k$ & $v_{f}, \gamma, \theta$ \\
\hline DiNenno $(2002)$ & $v(k)=v_{f} \exp \left(-\left(\frac{k}{\theta}\right)^{\gamma}\right)$ & $v_{f}, k_{j}, \gamma$ \\
\hline Tregenza $(1976)$ & $v(k)=v_{f}\left\{1-\exp \left(-\gamma\left(\frac{1}{k}-\frac{1}{k_{j}}\right)\right)\right\}$ & $v_{f}, \theta$ \\
\hline Weidmann $(1993)$ & $v(k)=v_{f} \exp \left(-\frac{k}{\theta}\right)$ & \\
\hline Rastogi et al. $(\overline{2013})$ & & \\
\hline Units: $k\left[\right.$ ped $\left./ m^{2}\right], v[m / s]$ & &
\end{tabular}

Table 2: Deterministic fundamental relationships - pedestrian traffic

Simulation-based fundamental relationships are predominantly obtained via cellular automaton models. For instance, Blue and Adler (1998) specified an unidirectional cellular automaton model that produces a speed-density relationship similar to the one proposed by Weidmann (1993). Few studies propose the specifications of fundamental relations derived from first principles (Flötteröd and Lämmel, 2015, Hoogendoorn et al. 2014).

The findings from several studies (Cheung and Lam, 1998; Daamen et al., 2005; Steffen and Seyfried, 2010), question the deterministic approach of the listed studies. They indeed report a significant scatter in the empirical speed-density relationship. The observed scatter is not possible to predict by the proposed deterministic models. Cheung and Lam (1998) have reported different distributions of the speed data observed for various ranges of density. In this study, speeds are less evenly distributed for lighter traffic conditions, which is explained by higher freedom that pedestrians have in controlling their movements. This indicates that in addition to density, other factors are likely to influence the speed of pedestrians. Weidmann (1993) has empirically shown that the trip purpose of pedestrians represents one of the relevant factors. According to this study free-flow speed of shopping pedestrians is $1.04 \mathrm{~m} / \mathrm{s}$, it is $1.45 \mathrm{~m} / \mathrm{s}$ for commuters and $0.99 \mathrm{~m} / \mathrm{s}$ for tourists. The speed of pedestrians appears to be affected by the age and the gender as well. According to Bowman and Vecellio (1994), the walking speed of 
pedestrians who are 60 years old and older is significantly lower than for the rest of the adult population. Weidmann (1993) has reported that children (under 12 years) are not capable of attaining the same speed as adults. According to the same study, walking speed of men is found to be $1.41 \mathrm{~m} / \mathrm{s}$, whereas for women it is lower $(1.27 \mathrm{~m} / \mathrm{s})$.

Existing models are not designed to capture these complex aspects. This is where our study makes a contribution. We propose a probabilistic speed-density relationship that is able to implicitly account for the heterogeneity of pedestrian flows.

\section{Foundation}

This section first presents the assumptions related to the quantities involved in our analysis, that is speed and density indicators at the microscopic level. We then present the derivation of the macroscopic relationships between the quantities. It serves as a basis for our modeling framework in Section 4 .

\subsection{Density and speed indicators}

The trajectory of pedestrian $i$ is a curve in space and time, that is

$$
p_{i}(t)=\left(x_{i}(t), y_{i}(t), t\right),
$$

where time $t$ spans the horizon of the analysis $\left[t_{0}, t_{f}\right]$, and $x_{i}(t)$ and $y_{i}(t)$ are the coordinates of the position of pedestrian $i$ at time $t$ in a given system of coordinates (typically, we express time in seconds, and use an orthonormal basis for the spatial dimensions).

In practice, the pedestrian trajectory data are collected through an appropriate tracking technology (e.g. Daamen and Hoogendoorn, 2003, Alahi et al. 2011). In this case, the time is discretized and the trajectory is described as a finite collection of triples

$$
p_{i s}=\left(x_{i s}, y_{i s}, t_{s}\right)
$$

where $t_{s}=\left(t_{0}, t_{1}, \ldots, t_{f}\right)$ corresponds to the available sample. We assume that the position of each pedestrian is known at each time $t_{s}$ of the discretization.

Different measurement methods have been proposed in the literature in order to obtain density and speed indicators from pedestrian trajectories. For a comprehensive analysis of several measurement methods and their influence on the fundamental diagram we refer to Zhang (2012). The measurement methods usually rely on a discretization scheme chosen arbitrarily, in both space and time (Seyfried et al. 2010, Daamen and Hoogendoorn 2003). The results may be highly sensitive to minor changes of discretization (Openshaw, 1983, Liddle et al., 2011). Our aim is to be as much independent from the aggregation level as possible and to preserve the heterogeneity of pedestrian population. We rely on a data-driven measurement method inspired by the one proposed by Steffen and Seyfried (2010). This method is based on the spatial discretization that is adjusted to the data through the use of Voronoi diagrams (Okabe et al. 2000).

The Voronoi space decomposition assigns a personal region to each pedestrian $i$, in such a way that each point in the personal region is closer to $i$ than to any other 
pedestrian, with respect of the Euclidean distance. In the presence of sampled data defined by (2), for each $s=0, \ldots, f$ and each pedestrian $i$, the personal region $V_{i s}$ is defined as

$$
V_{i s}=\left\{\left(\begin{array}{c}
x \\
y
\end{array}\right)\|\|\left(\begin{array}{c}
x \\
y
\end{array}\right)-\left(\begin{array}{c}
x_{i s} \\
y_{i s}
\end{array}\right)\left\|_{2} \leq\right\|\left(\begin{array}{c}
x \\
y
\end{array}\right)-\left(\begin{array}{c}
x_{j s} \\
y_{j s}
\end{array}\right) \|_{2}, \forall j\right\} .
$$

We assume that each point $(x, y)$ in space is associated with a unique Voronoi region at time $t_{s}$, corresponding to the region associated with pedestrian $i$, that is $V\left(x, y, t_{s}\right)=V_{i s}$. Note that if $(x, y)$ is exactly on the border between two or more regions, the unique region associated to it has to be arbitrarily defined.

Given the space discretization specified above, the density of pedestrians at position $(x, y)$ at time $t_{s}$ is

$$
k_{i s}=k\left(x, y, t_{s}\right)=\frac{1}{\left|V_{i s}\right|},
$$

where $V_{i s}$ is the unique Voronoi region that contains $(x, y)$ at time $t_{s}$, and $\left|V_{i s}\right|$ is the area of $V_{i s}$. The unit is the number of pedestrians per surface unit (typically, square meter). Figure 1 illustrates the map of density values obtained through the utilization of (4) for a given time instant. We refer to Steffen and Seyfried (2010) and Nikolić et al. (2014) for detailed discussions of this approach.

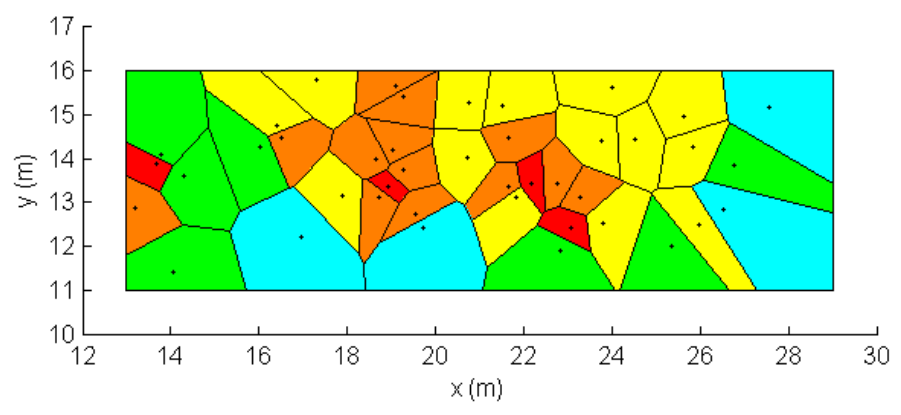

Figure 1: Voronoi-based density map

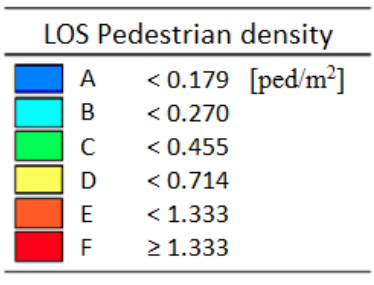

Table: Pedestrian walkway LOS density treshold values according to NCHRP (in SI units)

The velocity of pedestrian $i$ at time $t$ is given by

$$
\vec{v}_{i}(t)=v_{i}(t) \vec{d}_{i}(t),
$$

where $\vec{d}_{i}(t)$ is the (normalized) direction of pedestrian $i$ at time $t$ and $v_{i}(t)$ is the magnitude of the velocity vector, or speed. If the functions $x_{i}(t)$ and $y_{i}(t)$ in (1) are differentiable in $t$, it is defined as

$$
v_{i}(t)=\sqrt{\left(\frac{d x_{i}(t)}{d t}\right)^{2}+\left(\frac{d y_{i}(t)}{d t}\right)^{2}} .
$$

In the presence of discretized data, the speed is approximated using finite differences, for instance

$$
v_{i s}=\sqrt{\left(\frac{\Delta x_{i s}}{\Delta t}\right)^{2}+\left(\frac{\Delta y_{i s}}{\Delta t}\right)^{2}},
$$


where $\Delta x_{i s}=x_{i, s+1}-x_{i, s-1}, \Delta y_{i s}=y_{i, s+1}-y_{i, s-1}$, and $\Delta t=t_{s+1}-t_{s-1}$.

This definition assumes that the direction of the flow is unique at each point in time and space. It is therefore appropriate for the analysis of speed at an individual level. The definition may not result in the desired outcome if more aggregate characteristics are of interest, in particular if pedestrians in the same area do not walk in the same direction. For instance, if half of pedestrians walk in one direction and the rest in the opposite direction, but both with the same speed, their velocity vectors would cancel out at the aggregate level.

\subsection{Equilibrium speed-density relationships}

In order to derive the relationship between the indicators defined in Section 3.1 at the macroscopic level, we start from first principles. Similar to the approach presented in Hoogendoorn et al. (2014), the microscopic Social Force Model (SFM) proposed by Helbing and Molnar (1995) represents the basis of the derivation. The model explains the acceleration of pedestrian $i$ through the influence of neighboring pedestrians $j$

$$
\vec{a}_{i}=\frac{\vec{v}_{i}^{f}-\vec{v}_{i}}{\tau_{i}}-C_{i} \sum_{j} \exp \left(-\frac{R_{i j}}{B_{i}}\right) \vec{n}_{i j}\left(\lambda_{i}+\left(1-\lambda_{i}\right) \frac{1+\cos \left(\phi_{i j}\right)}{2}\right),
$$

where $\vec{v}_{i}^{f}$ is the desired velocity, $\vec{v}_{i}$ is the current velocity, $\tau_{i}$ is the relaxation time (the time needed to accelerate/decelerate to the desired velocity), $C_{i}$ is the interaction strength, $R_{i j}$ is the distance between pedestrians $i$ and $j, B_{i}$ is a scaling parameter, $\vec{n}_{i j}$ is the unit vector pointing from pedestrian $i$ to $j, \lambda_{i}$ denotes the anisotropy parameter and it ranges from 0 to 1 ( $\lambda_{i}=1$ implies isotropy), and $\phi_{i j}$ denotes the angle between the direction of $i$ and the position of $j$.

Consistently with many studies reported in the literature that are focused on the fundamental diagram (Section 2), we assume isotropic traffic conditions (identical properties in all directions). This leads to the model given as

$$
a_{i}=\frac{v_{i}^{f}-v_{i}}{\tau_{i}}-F_{i}^{r},
$$

where $F_{i}^{r}$ indicates the influence of the interaction between pedestrian $i$ with other pedestrians $j$ for isotropic conditions (the isotropic repulsive force). It is given as

$$
F_{i}^{r}=C_{i} \sum_{j} \exp \left(-\frac{R_{i j}}{B_{i}}\right) .
$$

A pedestrian $i$ experiences a greater repulsive force $F_{i}^{r}$ as other pedestrians are closer to $i$. This can be also reflected through a concept of a personal space associated with a pedestrian. Voronoi diagrams provide a way to derive a personal space "belonging" to a pedestrian, based on the position of $i$ and the positions of neighbors $j$ (see (3)). Given that the inverse of a personal space corresponds to density (as defined in (4)), the equation (9) can be rewritten as

$$
a_{i}=\frac{v_{i}^{f}-v_{i}}{\tau_{i}}-C_{i} k_{i} .
$$


Under the assumption that traffic conditions are stationary $\left(a_{i}=0\right)$, equation (11) results in the relationship between microscopic speed and density indicators

$$
v_{i}=v_{i}^{f}-\gamma_{i} k_{i}
$$

where $\gamma_{i}=\tau_{i} C_{i}$. To establish one-to-one speed-density relationship we further assume that pedestrian population is homogenous. This means that all the pedestrians have the same movement parameters (free-flow speed $v_{f}$ and sensitivity to congestion $\gamma$ ). In homogenous traffic under stationarity the distances between the pedestrians are also the same, so the densities are. Under these equilibrium conditions the fundamental speeddensity relationship is

$$
v_{e}=v_{e}(k)=v_{f}-\gamma k,
$$

where subscript 'e' denotes equilibrium. The resulting relationship correspond to the ones proposed by Older (1968), Navin and Wheeler (1969), Fruin (1971), Tanaboriboon et al. (1986) and Lam et al. (1995) (Table 2). It is also in agreement with the model of Hoogendoorn et al. (2014) for isotropic, homogenous and stationary traffic conditions.

Different SFM assumptions can lead to different fundamental relations under the equilibrium assumptions. If the closeness between pedestrians is assumed to affect the free-flow speed (e.g. the free-flow speed is an exponentially decreasing function of density), the resulting isotropic SFM can be specified as

$$
a_{i}=\frac{v_{i}^{f} \exp \left(-\left(\frac{k_{i}}{\theta_{i}}\right)^{\gamma_{i}}\right)-v_{i}}{\tau_{i}},
$$

where $\theta_{i}$ and $\gamma_{i}$ are the pedestrian-specific parameters. For pedestrian traffic at equilibrium (acceleration is zero and all pedestrians possess the same movement parameters) the relationship between speed and density resulting from (14) is given as

$$
v_{e}=v_{e}(k)=v_{f} \exp \left(-\left(\frac{k}{\theta}\right)^{\gamma}\right)
$$

This specification coincides with the speed-density relationship proposed by Tregenza (1976) (Table 2).

\section{Methodology}

We propose a probabilistic model to characterize speed-density relations. The model is derived by preserving the stationarity assumption and relaxing the homogeneity assumption of equilibrium relations derived in Section 3.2. In addition to the general modeling framework, we also present concrete suggestions for the operationalization of the derived model.

\subsection{Model derivation}

We assume that the speed of pedestrians is a random variable $(V)$, such that for each density level there is a distribution of speed values rather than one deterministic value. We assume that the following properties characterize this distribution: 
$P_{1}$ : The distribution of speed is continuous with positive support;

$P_{2}$ : The distribution of speed is unimodal.

The first property is in accordance with the physical characteristic of the speed, being that the speed is a continuous variable whose values cannot be negative. The second property is introduced with a purpose of maintaining the model parsimonious. Furthermore, we denote by $f_{\text {slow }}(\xi \mid k)$ the probability density function of the speed values lower than the equilibrium speed $v_{e}(k)$, and by $f_{\text {fast }}(\xi \mid k)$ the probability density function of the speed values greater than the equilibrium speed $v_{e}(k)$, both of which are conditional on density. We define the probability density function of the speed as

$$
f_{V}\left(\xi \mid v_{e}(k), \theta_{\text {slow }}(k), \theta_{\text {fast }}(k)\right)= \begin{cases}f_{\text {slow }}\left(\xi \mid v_{e}(k), \theta_{\text {slow }}(k)\right), & \xi \leq v_{e}(k) \\ f_{\text {fast }}\left(\xi \mid v_{e}(k), \theta_{\text {fast }}(k)\right), & \xi \geq v_{e}(k),\end{cases}
$$

where $\theta_{\text {slow }}(k)$ and $\theta_{\text {fast }}(k)$ are the density-dependent parameters that characterize slow, respectively fast, regime.

We do not give a specific formulation to individual random effects that may exist. Instead, we assume that individual random effects (i) are independent from other sources of randomness and do not correlate with any of the observed explanatory variable (e.g. density); (ii) do not affect the parameters' estimates and (iii) enter in an additive way the location parameters of the speed distribution.

For the illustration of the proposed methodology we need to specify the exact form of $v_{e}(k), f_{\text {slow }}\left(\xi \mid v_{e}(k), \theta_{\text {slow }}(k)\right)$ and $f_{\text {fast }}\left(\xi \mid v_{e}(k), \theta_{\text {fast }}(k)\right)$. In principle any specification may be chosen, as long as the required properties $P_{1}$ and $P_{2}$ are satisfied. We give some operational comments and propose concrete examples below.

\subsection{Operational comments}

The proposed specification of the speed-density relationship (16) does not satisfy the properties of the $C^{1}$ class functions. The maximum likelihood estimation of its parameters may therefore involve complex non-smooth optimization algorithms. Also, the value of $v_{e}(k)$ is not observed and has to be modeled. To address these issues we adopt a mixing technique, where $v_{e}(k)$ is assumed to be distributed according to a parametric distribution $f_{v_{e}(k)}\left(\zeta ; \theta_{v_{e}}(k)\right)$ with density-dependent parameters $\theta_{v_{e}}(k)$.

The resulting likelihood function is

$$
f_{\text {PedProb-vk }}\left(\xi \mid k ; \theta_{\text {slow }}(k), \theta_{\text {fast }}(k), \theta_{v_{e}}(k)\right)=\int_{\zeta=0}^{\infty} f_{V}\left(\xi \mid \zeta ; \theta_{\text {slow }}(k), \theta_{\text {fast }}(k)\right) f_{v_{e}(k)}\left(\zeta ; \theta_{v_{e}}(k)\right) d \zeta,
$$

where $f_{V}\left(\xi \mid \zeta ; \theta_{\text {slow }}(k), \theta_{\text {fast }}(k)\right)$ is defined by (16). The model is called PedProb-vk, which stands for Pedestrian Probabilistic speed (v) - density (k) relationship.

\subsection{Exemplary specification}

We suggest a linear model for the distribution of the speed of the slow component, since it is well adjusted to impose the lower bound of the distribution at zero

$$
f_{\text {slow }}\left(\xi \mid v_{e}(k), \alpha_{k}, \beta_{k}\right)=\frac{\beta_{k}-\alpha_{k}}{v_{e}(k)} \xi+\alpha_{k},
$$


where $0 \leq \xi \leq v_{e}(k)$, and $\alpha_{k} \geq 0$ and $\beta_{k} \geq 0$ are parameters dependent on $k$. They are such that $f_{\text {slow }}(0 \mid k)=\alpha_{k}$ and $f_{\text {slow }}\left(v_{e}(k) \mid k\right)=\beta_{k}$. We adopt a simple specification for the parameters $\alpha_{k}, \beta_{k}$. We assume that they depend on the density $k$ in the following way

$$
\alpha_{k}\left(a_{\alpha}, b_{\alpha}\right)=a_{\alpha} k+b_{\alpha},
$$

and

$$
\beta_{k}\left(a_{\beta}, b_{\beta}\right)=a_{\beta} k+b_{\beta}
$$

where $a_{\alpha} \geq 0, b_{\alpha} \geq 0, a_{\beta} \geq 0$ and $b_{\beta} \geq 0$. They are such that $\alpha_{0}=b_{\alpha}$ and $\beta_{0}=b_{\beta}$.

We propose an exponential model for the distribution of the speed of the fast component

$$
f_{\text {fast }}\left(\xi \mid v_{e}(k), \beta_{k}, \lambda\right)=\exp \left(-\lambda \xi+\log \left(\beta_{k}\right)+\lambda v_{e}(k)\right),
$$

where $\xi \geq v_{e}(k), v_{e}(k)$ and $\beta_{k}$ are defined as above, and $\lambda \geq 0$ is an additional parameter, defining the rate of the exponential distribution. The choice of the exponential specification is well suited to avoid any arbitrariness in imposing the upper bound, while preventing arbitrary high values at the same time. The normalizing constant is in this example equal to $\frac{\alpha_{k}+\beta_{k}}{2} v_{e}(k)+\frac{\beta_{k}}{\lambda}$.

Note that, the value determined by $v_{e}(k)$ represents the mode of the distribution $\left(\frac{d f_{v}\left(\xi \mid v_{e}(k), \alpha_{k}, \beta_{k}, \lambda\right)}{d \xi}=0\right.$ when $\left.\xi(k)=v_{e}(k)\right)$. If $\xi=v_{e}(k)$, the values of $f_{\text {slow }}(\xi \mid k)$ and $f_{\text {fast }}(\xi \mid k)$ coincide, and are both equal to $\beta_{k}$. When $k=0$, then $f_{\text {slow }}(0 \mid k)=b_{\alpha}, f_{\text {slow }}\left(v_{e}(k) \mid k\right)=$ $f_{\text {fast }}\left(v_{e}(k) \mid k\right)=b_{\beta}$ and $f_{\text {fast }}(\xi \mid k)<b_{\beta}$ if $\xi>v_{e}(k)$. When $k \rightarrow \infty$, then $f_{\text {slow }}(0 \mid k)=f_{\text {fast }}(0 \mid k) \rightarrow$ $\infty$ and $f_{\text {fast }}(\xi \mid k) \rightarrow 0$. The parameters characterizing the distribution of each component are illustrated in Figure 2.

We use a symmetric triangular distribution for mixing defined on the interval $\left[\bar{v}_{e}(k)-\right.$ $\left.\sigma(k), \bar{v}_{e}(k)+\sigma(k)\right]$, where $\bar{v}_{e}(k)$ is the mean of the distribution and $\sigma(k)=\frac{1}{1+\exp (\eta)} \bar{v}_{e}(k)$

$$
f_{v_{e}(k)}\left(\zeta ; \bar{v}_{e}(k), \sigma(k)\right)=\left\{\begin{array}{lr}
\frac{\zeta-\bar{v}_{e}(k)+\sigma(k)}{\sigma(k)^{2}}, & \bar{v}_{e}(k)-\sigma(k) \leq \zeta \leq \bar{v}_{e}(k) \\
\frac{\bar{v}_{e}(k)+\sigma(k)-\zeta}{\sigma(k)^{2}}, & \bar{v}_{e}(k)<\zeta \leq \bar{v}_{e}(k)+\sigma(k) \\
0, & \zeta<\bar{v}_{e}(k)-\sigma(k) \text { or } \zeta>\bar{v}_{e}(k)+\sigma(k) .
\end{array}\right.
$$

The choice of this specific distribution is motivated by the simple closed form of its probability density function and cumulative density function. The proposition for the specification of $\sigma(k)$ insures that the property $P_{1}$ is satisfied. The specification of $\bar{v}_{e}(k)$ is typically a deterministic speed-density relationship such as those presented in Section 3.2 .

Putting everything together, the distribution of the speed in our example is given by

$$
f_{\text {PedProb-vk }}\left(\xi \mid k ; \alpha_{k}, \beta_{k}, \lambda, \bar{v}_{e}(k), \sigma(k)\right)=\int_{\zeta=\bar{v}_{e}(k)-\sigma(k)}^{\bar{v}_{e}(k)+\sigma(k)} f_{V}\left(\xi \mid \zeta ; \alpha_{k}, \beta_{k}, \lambda\right) f_{v_{e}(k)}\left(\zeta ; \bar{v}_{e}(k), \sigma(k)\right) d \zeta,
$$

where the distributions of the slow and the fast component are defined by (18), respectively (21), and $f_{v_{e}(k)}\left(\zeta ; \bar{v}_{e}(k), \sigma(k)\right)$ is defined by (22). For this specification, the integral (23) does not have a closed-form, and numerical integration or Monte-Carlo simulation is required. However, other specifications of $f_{v_{e}(k)}\left(\zeta ; \theta_{v_{e}}(k)\right), f_{\text {slow }}\left(\xi \mid v_{e}(k), \theta_{\text {slow }}(k)\right)$ and $f_{\text {fast }}\left(\xi \mid v_{e}(k), \theta_{\text {fast }}(k)\right)$ may lead to a closed-form formulation, as illustrated in Appendix. 


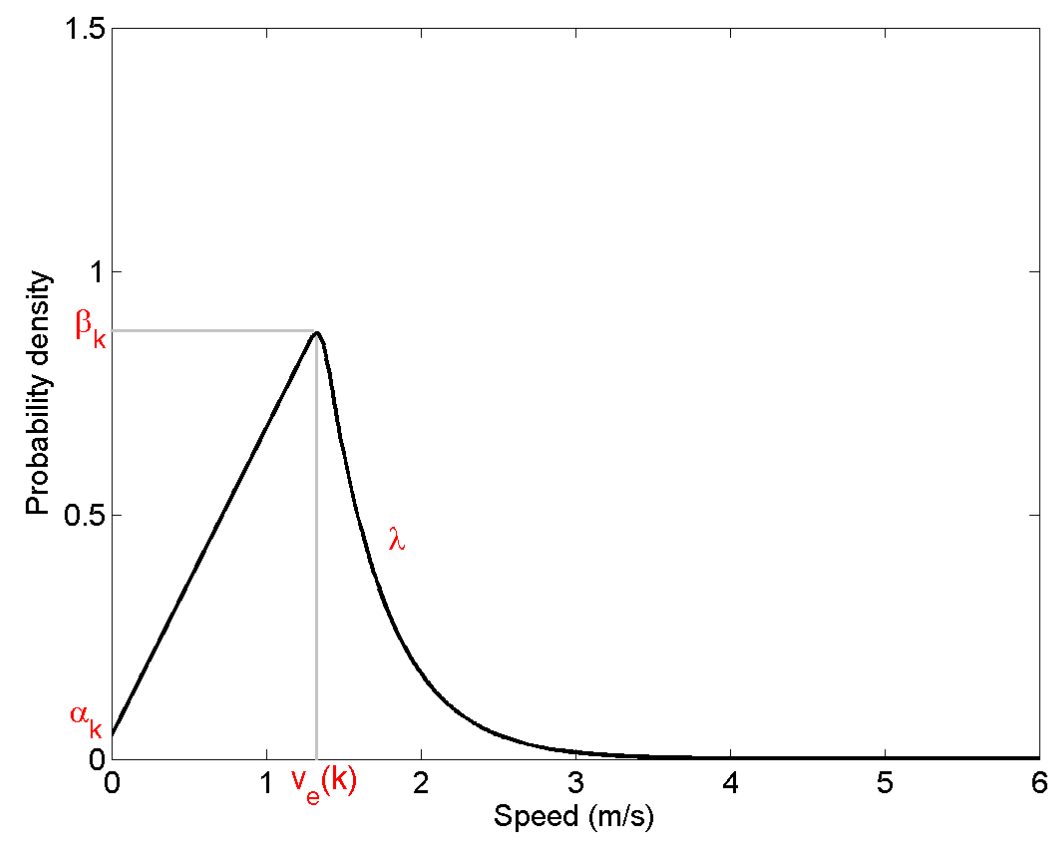

Figure 2: Illustration of the model - one density level

We emphasize that the proposed specification is merely one possibility, and the framework is general and other specifications are possible (e.g. different functional forms of $v_{e}(k), f_{v_{e}(k)}\left(\zeta ; \theta_{v_{e}}(k)\right), f_{\text {slow }}\left(\xi \mid v_{e}(k), \theta_{\text {slow }}(k)\right)$ and $f_{\text {fast }}\left(\xi \mid v_{e}(k), \theta_{\text {fast }}(k)\right)$, different smoothing strategies, etc). To illustrate and validate our approach, we perform next the analysis on two case studies (Section 6. Section 7).

\subsection{Estimation procedure}

For the estimation of the model parameters, we use quasi-maximum likelihood estimation. 1 As each pedestrian generates several piece of data, there is serial correlation among these observation. If ignored, the unobserved/ unmodeled covariance among the observations of a same individual in the estimation procedure leads to consistent but inefficient estimates (Gourieroux et al., 1984, Trognon, 1987; Wooldridge, 2010). This holds as long as the first order moment of the distribution of the endogenous variable is not affected by its higher order moments (Gourieroux et al., 1984), i.e. independence between mean and higher moments. In this case, the Rao-Cramer bound is not reached, and a block bootstrap method must be used for the calculation of the standard errors of the estimates (Hall et al., 1995; Davison and Hinkley, 1997; Davidson and MacKinnon,

\footnotetext{
${ }^{1}$ Quasi-maximum likelihood estimation differs from full information maximum likelihood estimation in the sense that it is necessary to explicitly specify the covariance structure for the latter.
} 
2004). It is also convenient to assume that there is no correlation across pedestrians, and that the observations of one and the same pedestrian are serially correlated due to unobserved time invariant specific effects. Bootstrap replications are thus based on the sampling over blocks, where each block contains the series of the observations of one individual. We adopt these assumptions in the case studies presented below.

\section{Case studies and empirical investigation}

The motivation of this research comes from the analysis of two real datasets, that we use below to illustrate and validate our approach.

\subsection{Lausanne train station}

The first dataset is collected in a pedestrian underpass of the train station of Lausanne, Switzerland. Figure 3 shows the layout of the studied area. It covers approximately $685 \mathrm{~m}^{2}$. The underpass is frequently used especially during the morning and afternoon peak hours since it connects the exterior of the train station to the main platforms. It also acts as a connection between mostly residential south and the center of the city in the north.

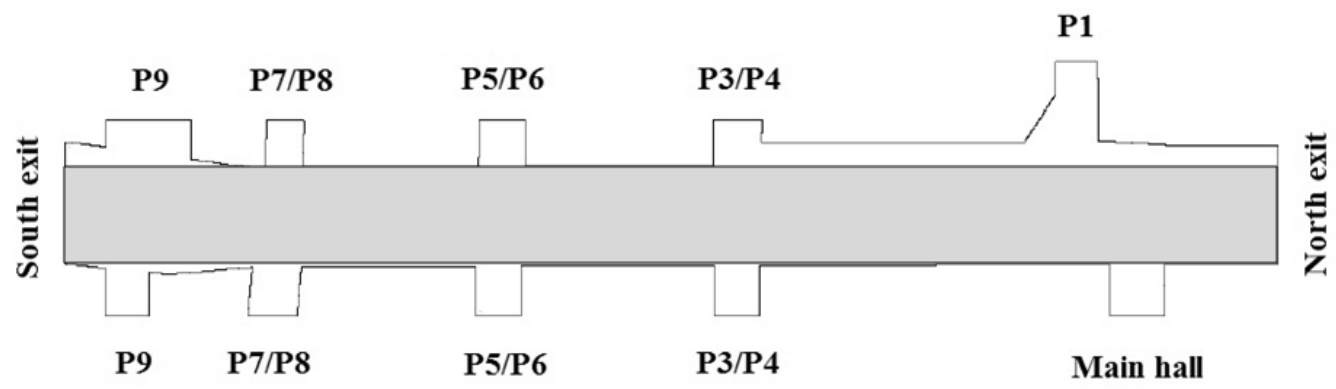

Figure 3: Lausanne train station - pedestrian underpass West

To collect the raw data, a large-scale network of smart sensors has been deployed in the station. The underlying technology is based on infrared and depth sensors that detect silhouettes and track each pedestrian in the scene covered by the network. The tracking engine uses a sparsity driven framework (Alahi et al. 2011, 2014) to link detected pedestrians over the network of sensors.

It results in a dataset of 25,603 trajectories, collected during a time period between 07:00 and 08:00 on February 12,13,14, 15 and 18, 2013. The temporal resolution of every trajectory ranges from 10 to 25 points per second and it has been processed to obtain the position of every pedestrian in the scene at every second. The average length of the trajectories is 78 meters and the duration of a pedestrians' stay in the underpass ranges from 15 seconds to 2.2 minutes.

Note that we have selected only trajectories collected in the shaded area shown in Figure 3, referring to a corridor. The trajectories from the ramps and stairs (denoted 
as P1-P9) are not considered in this study. Indeed, as explained by Daamen (2004) and Weidmann (1993), the walking behavior and, therefore, the speed-density relationship, varies with the type of infrastructure.

In the rest of the paper, we refer to this case study as the Lausanne case study.

\subsection{Controlled experiment}

The second set of data has been collected during a controlled experiment at the Technical University of Delft in the Netherlands (Daamen and Hoogendoorn 2003). The individuals participating in the experiment were instructed to walk along a corridor that is 10 meters long and 4 meters wide, at a normal speed, and to pass through a bottleneck of 1 meter in width (see Figure 4 where individuals walk from right to left).

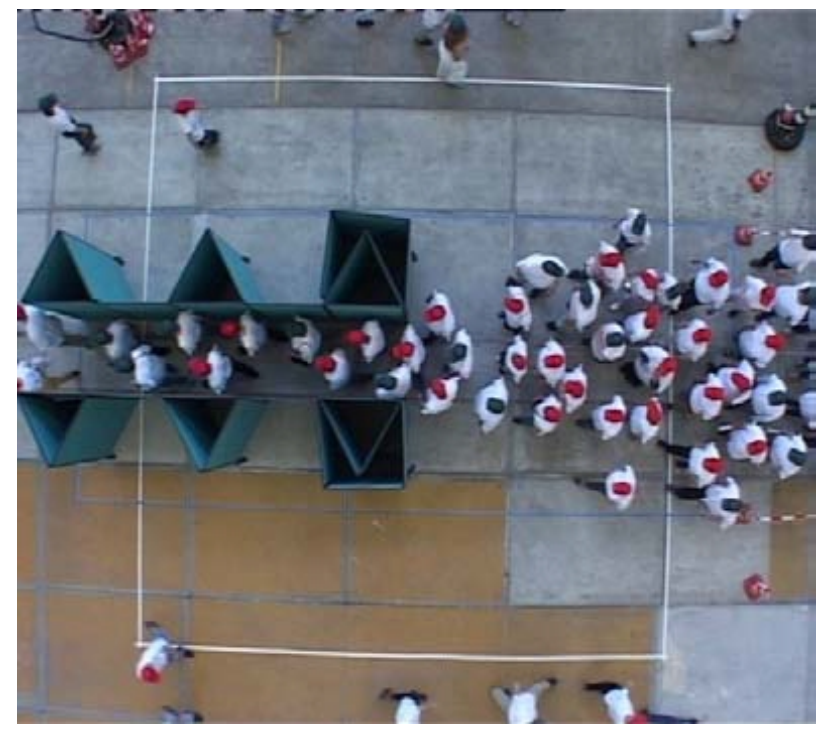

Figure 4: Narrow bottleneck experiment (Daamen and Hoogendoorn, 2003)

The scene was filmed from the top by digital cameras. The individual trajectories were extracted from the digital video sequences.

The experiment lasted about 15 minutes. A total of 1,123 trajectories were collected, where the position of each individual is available every 0.1 second. The average length of the trajectories is similar inside and upstream of the bottleneck and it is approximately 5 meters. The average travel time of the trajectories upstream of the bottleneck is 10 seconds, whereas inside the bottleneck it is lower (approximately 5 seconds).

Note that we have selected trajectories collected in the rectangular area (5 meters long and 4 meters wide) upstream of the bottleneck (Figure 44. As explained by Duives et al. (2014, Figure 4), this is where the variability is observed.

In the rest of the paper, we refer to this case study as the Delft case study. 


\subsection{Empirical analysis}

The speed-density profiles corresponding to the Lausanne and the Delft case studies are obtained from the measurement method presented in Section 3.1. In Figure 5 each circle corresponds to one observation, that is, one pedestrian at one specific time in the horizon. The $x$ coordinate of the circle corresponds to the density, calculated from (4), and its $y$ coordinate corresponds to the speed calculated from (7).

Figure 5 a plots 270,291 observations corresponding to the peak hour of February 12, 2013 for the Lausanne case study. The same pattern was observed on any weekday. Figure 5 b plots 119,156 observations for the Delft case study.

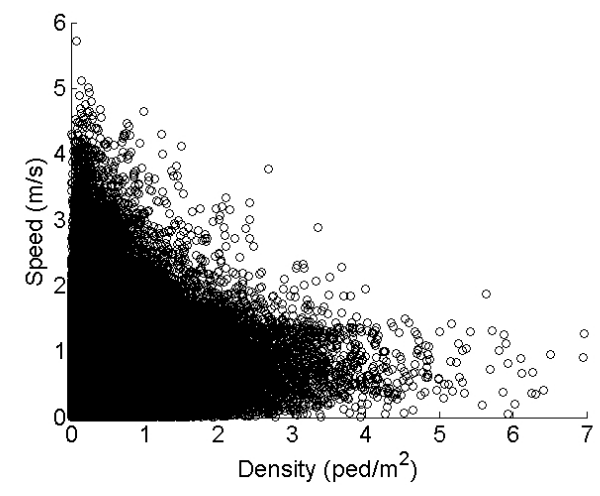

(a) Lausanne case study

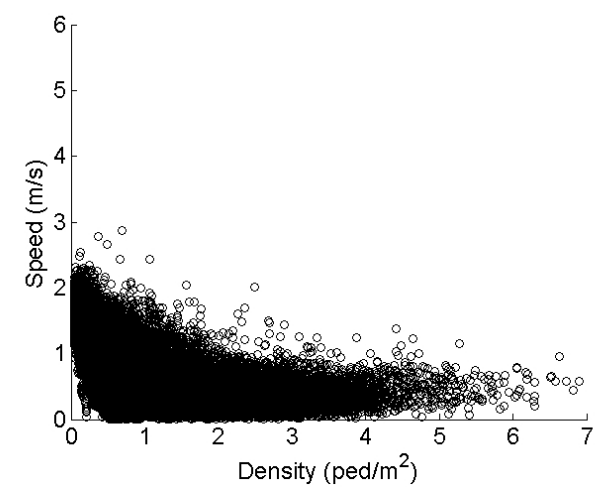

(b) Delft case study

Figure 5: Speed-density profiles

A high scattering is observed in both cases (Figure 5). The density ranges from 0 to approximately 7 pedestrians per square meter. In the Lausanne case, the speed ranges from 0 to 5.72 meters per second (that is about $21 \mathrm{~km} / \mathrm{h}$ ), and $99 \%$ of the observations are between 0 and 2.42 meters per second (that is about $9 \mathrm{~km} / \mathrm{h}$ ). In the Delft case, the speed ranges from 0 to 2.87 meters per second (that is about $10 \mathrm{~km} / \mathrm{h}$ ). The difference in the speed distribution is attributed to the controlled nature of the experiment in Delft, where individuals where instructed to walk at normal speed, resulting to a lower variance compared to Lausanne, where no instruction was given. For the same reason, low speeds where not observed at low density in Delft, contrarily to Lausanne.

To investigate this data in more details, the speed distributions at various density levels are presented in Figures 6 and 7 for the Lausanne and Delft case study, respectively. In both cases, a higher level of variability is noticeable at lower densities, compared to higher densities where the distribution of speed is less spread and shifted towards lower values.

The deterministic models for the speed-density relationship proposed in the literature (Section 2.2) appear to be inadequate for representing the observed patterns. Clearly, density is not the only factor influencing pedestrians' speed. We emphasize that there are different sources of uncertainty that can contribute to the observed pattern: (i) the 
variability due to the nature of the system (e.g. heterogeneity of behavior, interaction of behaviors such as people walking in groups, the existence of multi-directional flows that are more chaotic) and (ii) measurement errors (e.g. discretization and measurement noise, technological issues). The Voronoi based approach is completely data-driven and designed to minimize the spatial discretization errors. Given the type of the available data, these sources are not separable from each other. Instead, the proposed probabilistic model for the speed-density relationship accounts for the whole uncertainty at once.

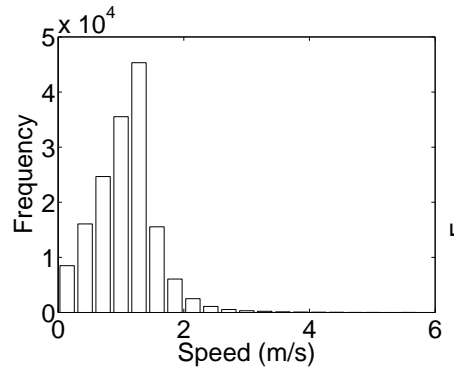

(a) $\mathrm{k}<0.1 \mathrm{ped} / \mathrm{m}^{2}$

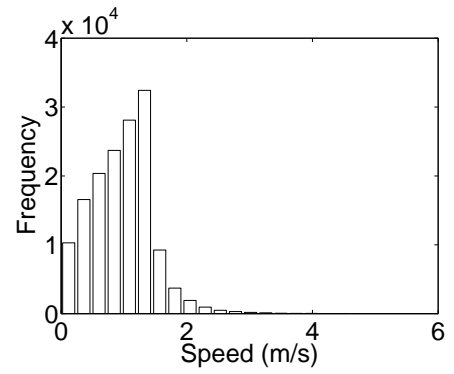

(d) $\mathrm{k}=0.6 \mathrm{ped} / \mathrm{m}^{2}$

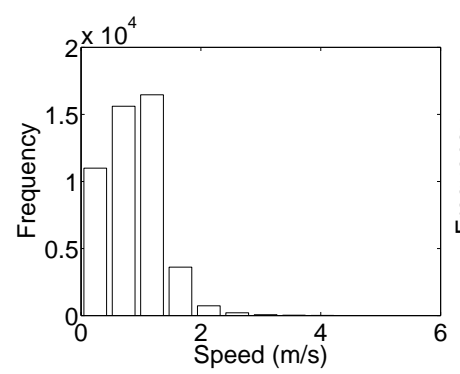

(g) $\mathrm{k}=1.2 \mathrm{ped} / \mathrm{m}^{2}$

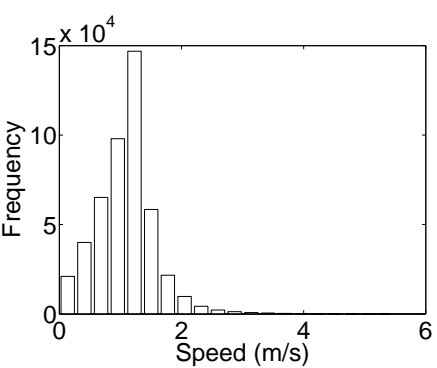

(b) $\mathrm{k}=0.2 \mathrm{ped} / \mathrm{m}^{2}$

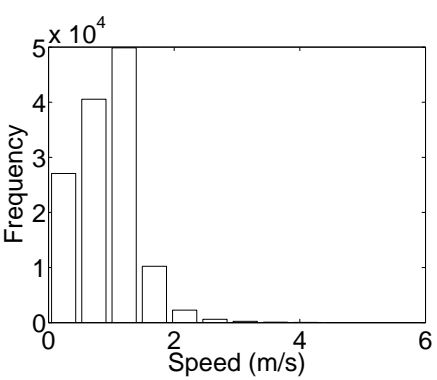

(e) $\mathrm{k}=0.8 \mathrm{ped} / \mathrm{m}^{2}$

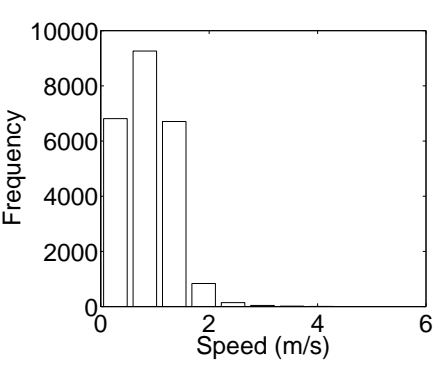

(h) $\mathrm{k}=1.5 \mathrm{ped} / \mathrm{m}^{2}$

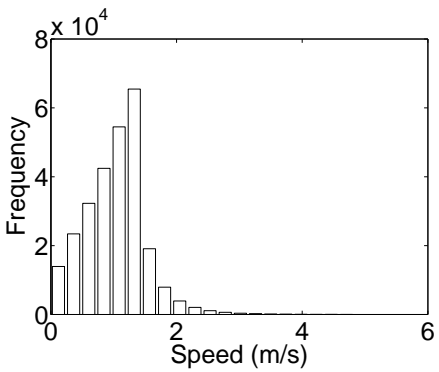

(c) $\mathrm{k}=0.4 \mathrm{ped} / \mathrm{m}^{2}$

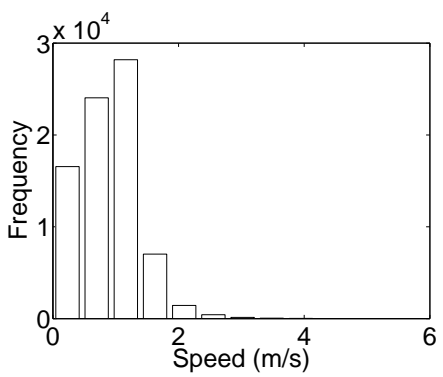

(f) $\mathrm{k}=1 \mathrm{ped} / \mathrm{m}^{2}$

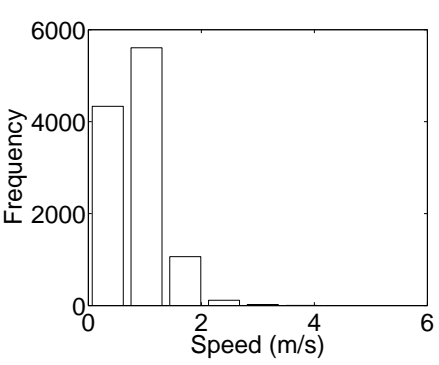

(i) $\mathrm{k}=2 \mathrm{ped} / \mathrm{m}^{2}$

Figure 6: Speed distributions for different density levels - Lausanne case study 


\section{Case study: Lausanne}

We illustrate and validate now the model on the Lausanne dataset, introduced in Section 5.1. The dataset used for the estimation consists of 1,269,393 pairwise speeddensity observations corresponding to the peak hour of February 12, 13, 14, 15 and 18, 2013.

The descriptive statistics of the estimation dataset are presented in Table 3 . The dataset is categorized according to six levels of service (LOS) proposed by Fruin (1971) for pedestrian facilities, labeled from A to F. Table 3 shows that the largest part of the observations falls below the LOS F. Actually, $99 \%$ of the observations are below 2.06 $\mathrm{ped} / \mathrm{m}^{2}$.

\begin{tabular}{|l|c|}
\hline Level Of Service & Number of observations \\
\hline $\mathrm{A}\left(\mathrm{k} \leq 0.31 \mathrm{ped} / \mathrm{m}^{2}\right)$ & 644546 \\
\hline $\mathrm{B}\left(\mathrm{k} \in\left(0.31-0.43 \mathrm{ped} / \mathrm{m}^{2}\right]\right)$ & 174116 \\
\hline $\mathrm{C}\left(\mathrm{k} \in\left(0.43-0.71 \mathrm{ped} / \mathrm{m}^{2}\right]\right)$ & 229808 \\
\hline $\mathrm{D}\left(\mathrm{k} \in\left(0.71-1.11 \mathrm{ped} / \mathrm{m}^{2}\right]\right)$ & 133812 \\
\hline $\mathrm{E}\left(\mathrm{k} \in\left(1.11-2.17 \mathrm{ped} / \mathrm{m}^{2}\right]\right)$ & 76725 \\
\hline $\mathrm{F}\left(\mathrm{k}>2.17 \mathrm{ped} / \mathrm{m}^{2}\right)$ & 10386 \\
\hline
\end{tabular}

Table 3: Estimation data classified according to LOS Fruin 1971) - Lausanne case study

The estimation results for the model (23) presented in Section 4.3 are shown in Table 4 The number of iterations (200) for the block bootstrap has been determined by computing bootstrap approximation of the mean square error of the estimates (Ross, 2013b. Section 8.3). The parameter $\bar{v}_{e}(k)$ is specified in the model (13).

All estimates have the expected sign and value. The results also show the low standard errors of all parameters and their statistical significance (t-test) at a usual significance level (0.05) ${ }^{2}$

The positive sign of the parameter $a_{\alpha}$ shows that $\alpha_{k}$, that is the likelihood of low speeds, increases with density. Similarly, the positive sign of the parameter $a_{\beta}$ shows that $\beta_{k}$, that is the likelihood of the mode of the speed distribution, also increases with density.

The signs and the estimated values of the parameters $v_{f}$ and $\gamma$ are consistent with the ones reported in the literature (see Section 2 ) and with the trend observed in the data. The low variance of the parameter $\eta$ is as expected, since the mixing distribution (22) is introduced for the smoothing purposes.

Various alternative specifications of the model have been investigated in order to represent the speed-density relationship. The first alternative specification (denoted as the specification A), assumes the Kumaraswamy distribution of speed values (e.g. Nikolić et al. (2014)). In the second specification (denoted as the specification B), the speed

\footnotetext{
${ }^{2}$ The high significance of the parameters can also be attributed to the large number of observations used for the model estimation.
} 


\begin{tabular}{cccc}
\hline Parameter & Value & Std err (block bootstrap) & t-test \\
\hline$a_{\alpha}$ & 0.0393 & $4.78 e^{-03}$ & 8.52 \\
$b_{\alpha}$ & 0.00708 & $8.85 e^{-04}$ & 7.87 \\
$a_{\beta}$ & 0.00487 & $2.41 e^{-03}$ & 2.10 \\
$b_{\beta}$ & 0.142 & $3.71 e^{-03}$ & 38.4 \\
$\lambda$ & 3.53 & $1.70 e^{-03}$ & $2.07 e^{03}$ \\
$v_{f}$ & 1.29 & $2.85 e^{-03}$ & $4.57 e^{02}$ \\
$\gamma$ & 0.0512 & $3.85 e^{-03}$ & 13.4 \\
$\eta$ & 3.48 & $1.25 e^{-03}$ & $2.77 e^{03}$ \\
\hline $\log \mathcal{L}$ & -783942.897 & & \\
Number of parameters & 8 & & \\
Number of observations & 1269393 & & \\
\hline
\end{tabular}

Table 4: Estimation results - Lausanne case study

values are assumed to be generated from the Rayleigh distribution. The third specification (denoted as the specification $\mathrm{C}$ ), assumes the Weibull distribution of speed values. In all cases the parameters of the distributions are allowed to vary with density. Also, the alternative specification of PedProb-vk is considered (denoted as the specification D), where we tried for $\bar{v}_{e}(k)$ the specification proposed by Weidmann (1993) (Table 2). All alternative specifications resulted in a poorer fit with respect to PedProb-vk, when compared by the means of the Bayesian information criterion - BIC (Wasserman, 2000):

$$
B I C=-2 \cdot \log \mathcal{L}+r \cdot \log (n)
$$

where $n$ is the nuber of the observations, $r$ is the number of parameters to be estimated and $\mathcal{L}$ is the value of the likelihood function of the model. The model with the lowest BIC is preferred. Indeed, the BIC values shown in Table 5 suggest that the PedProb-vk represents the best compromise between the model accuracy and simplicity among all evaluated specifications.

\begin{tabular}{|c|c|c|c|c|c|}
\hline Model specification & A & B & C & D & PedProb-vk \\
\hline $\log \mathcal{L}$ & -891878.142 & -897336.414 & -895420.690 & -845079.532 & -783942.897 \\
\hline \# parameters & 8 & 3 & 4 & 9 & 8 \\
\hline \# observations & 1269393 & 1269393 & 1269393 & 1269393 & 1269393 \\
\hline$B I C$ & 1783868.715 & 1794678.828 & 1790897.596 & 1690285.550 & 1567998.226 \\
\hline
\end{tabular}

Table 5: Goodness of Fit (BIC) - Lausanne case study

\subsection{Comparison with deterministic models}

The performance of the proposed probabilistic model at the aggregate level is compared with the deterministic models proposed in the literature (Table 2). We have estimated the parameters of these models using linear regression on our dataset. 
For PedProb-vk, the average speed is given by

$$
\bar{v}_{\text {PedProb-vk }}(k)=\int_{0}^{\infty} \xi f_{\text {PedProb-vk }}\left(\xi \mid k ; \alpha_{k}, \beta_{k}, \lambda, \bar{v}_{e}(k), \sigma(k)\right) d \xi .
$$

In (25) $f_{\text {PedProb-vk }}\left(\xi \mid k ; \alpha_{k}, \beta_{k}, \lambda, \bar{v}_{e}(k), \sigma(k)\right)$ refers to mixture distribution given by (23), with the parameters described in Table 4 .

Figure 8 shows a comparison among four deterministic models, the aggregate speed calculated from PedProb-vk, and the observed values. The analysis is performed for density levels ranging from 0 to $2.06 \mathrm{ped} / \mathrm{m}^{2}$, corresponding to $99 \%$ of the observed values. The goodness of fit is reported in Table 6 , where

$$
M S E=\frac{1}{m} \sum_{\ell=1}^{m}\left(\bar{v}_{\text {model }}\left(k_{\ell}\right)-\bar{v}_{\text {data }}\left(k_{\ell}\right)\right)^{2},
$$

where $m=22$ and $k_{\ell}=2.06(\ell-1) /(m-1)$. The value $\bar{v}_{\text {data }}\left(k_{\ell}\right)$ is the average of the observed speeds corresponding to densities ranging from $\left(k_{\ell}+k_{\ell-1}\right) / 2$ and $\left(k_{\ell}+k_{\ell+1}\right) / 2$. To account for the number of parameters in the model relative to the sample size we also report the adjusted coefficient of determination $\left(\bar{R}^{2}\right)$ (Hair et al. 2009, p. 170) in Table 6 Although the PedProb-vk has been calibrated at the disaggregate level, it is

\begin{tabular}{|c|c|c|c|c|c|}
\hline Model & Weidmann (1993) & Tregenza (1976) & Rastogi et al. (2013) & Linear & PedProb-vk \\
\hline$M S E$ & $6.69 e^{-03}$ & $1.82 e^{-\overline{5}}$ & $1.90 e^{-03}$ & $1.87 e^{-03}$ & $1.27 e^{-03}$ \\
\hline $\bar{R}^{2}$ & $7.67 e^{-03}$ & $7.31 e^{-01}$ & $7.18 e^{-01}$ & $7.22 e^{-01}$ & $8.12 e^{-01}$ \\
\hline
\end{tabular}
interesting to observe that it achieves the best fit at the aggregate level.

Table 6: Goodness of Fit (aggregate level) - Lausanne case study

\subsection{Kolmogorov-Smirnov validation}

The validation is performed by comparing the distribution functions of the estimated model, and the empirical distributions from the dataset. The analysis is carried out at different density levels. Figure 9 shows the probability density functions of the model (model pdf) and the data (empirical histogram) at the same density level. The corresponding cumulative density functions (model cdf and empirical cdf) are plotted in Figure 10. Qualitatively, the match between the two is pretty satisfactory.

For the quantitative analysis, we use the Kolmogorov-Smirnov probability distance metric (Massey, 1951)

$$
D_{k}=\max _{v}\left|F_{\text {model }}(v \mid k)-F_{\text {data }}(v \mid k)\right|,
$$

where $F_{\text {model }}(v \mid k)$ corresponds to the model cdf and $F_{\text {data }}(v \mid k)$ to the empirical cdf. This metric represents the maximum value of the absolute vertical difference between the two cumulative distribution functions. It is reported in Figure 11a.

We calculate the $p$-value of the Kolmogorov-Smirnov statistic using simulation (Ross, 2013a p. 257), with 100 simulation runs. The model (23) is simulated using the rejection method (Ross, 2013b, Section 5.2) on draws from a Rayleigh distribution. The results 
are shown in Figure 11b. They suggest that there is no evidence in the data to reject PedProb-vk at significance level 0.05 for all levels of density except maybe for the one corresponding to densities close to zero.

\subsection{Specification test}

In order to test the robustness of the proposed specification, we have performed the validation that consists in splitting the dataset into two subsets. The model is re-estimated on one subset and the remaining data, unused for estimation, is used for validation purposes. The procedure consisting of the following steps is repeated 100 times:

1. A sample of $80 \%$ of pairwise speed-density observations is selected using simple random selection.

2. The parameters of the model are estimated using the generated sample.

3. The Kolmogorov-Smirnov statistic $D_{k}(27)$ is calculated to compare the estimated model and the data on the remaining $20 \%$ of the dataset.

In Figure 12a we compare the value of $D_{k}$ calculated on the full dataset (in dashed line) with the values calculated with the above mentioned procedure. The 100 values are summarized using a box plot at each level of density. These results are satisfactory. The specification is robust and no overfitting is detected.

To be more precise, we also calculate the $p$-value for each value of $D_{k}$ calculated with the above mentioned procedure. For this purpose we use simulation (Ross, 2013a p. $252)$, with 100 simulation runs. The box plot of the estimated $p$-values are shown in Figure $12 \mathrm{~b}$. The results do not allow to reject the hypothesis that the data and the model follow the same distribution, at a usual level of significance.

\section{Case study: Delft}

In this section we illustrate and validate the model on the Delft dataset, described in Section 5.2

The dataset used for estimation consist of 119,156 pairwise speed-density values observed upstream of the bottleneck. The data has been classified according to the LOS standard of Fruin (1971), showing that now the majority of the observations corresponds to the LOS C, D, E and F (Table 7). Consequently $99 \%$ of the data is below the density value of $3.9 \mathrm{ped} / \mathrm{m}^{2}$. This is as expected, given the existence of flow constraint (in the form of a narrow bottleneck) that in this case causes congestion upstream of the bottleneck.

The parameters of the model (23) have been estimated, where $\bar{v}_{e}(k)$ is specified by the model (15). The estimation results are shown in Table 8 . The sign and the magnitude of the parameters are as expected. The standard errors of the parameters were computed via block bootstrap method (see Section 4.4). The results also indicate a high significance of the estimated values. 


\begin{tabular}{|l|c|}
\hline Level Of Service & Number of observations \\
\hline $\mathrm{A}\left(\mathrm{k} \leq 0.31 \mathrm{ped} / \mathrm{m}^{2}\right)$ & 9288 \\
\hline $\mathrm{B}\left(\mathrm{k} \in\left(0.31-0.43 \mathrm{ped} / \mathrm{m}^{2}\right]\right)$ & 6967 \\
\hline $\mathrm{C}\left(\mathrm{k} \in\left(0.43-0.71 \mathrm{ped} / \mathrm{m}^{2}\right]\right)$ & 20497 \\
\hline $\mathrm{D}\left(\mathrm{k} \in\left(0.71-1.11 \mathrm{ped} / \mathrm{m}^{2}\right]\right)$ & 21540 \\
\hline $\mathrm{E}\left(\mathrm{k} \in\left(1.11-2.17 \mathrm{ped} / \mathrm{m}^{2}\right]\right)$ & 37114 \\
\hline $\mathrm{F}\left(\mathrm{k}>2.17 \mathrm{ped} / \mathrm{m}^{2}\right)$ & 23750 \\
\hline
\end{tabular}

Table 7: Estimation data classified according to LOS Fruin, 1971) - Delft case study

\begin{tabular}{cccc}
\hline Parameter & Value & Std err (block bootstrap) & t-test \\
\hline$a_{\alpha}$ & 0.165 & $6.62 e^{-02}$ & 2.08 \\
$b_{\alpha}$ & 0.244 & $6.97 e^{-02}$ & 3.11 \\
$a_{\beta}$ & 0.166 & $6.85 e^{-02}$ & 2.49 \\
$b_{\beta}$ & 0.965 & $5.97 e^{-02}$ & 16.4 \\
$\lambda$ & 6.90 & $1.41 e^{-02}$ & $4.89 e^{02}$ \\
$v_{f}$ & 1.87 & $4.92 e^{-02}$ & 38.1 \\
$\theta$ & 1.13 & $6.03 e^{-02}$ & 18.3 \\
$\gamma$ & 0.545 & $4.31 e^{-02}$ & 13.1 \\
$\eta$ & 3.29 & $3.28 e^{-02}$ & $1.00 e^{02}$ \\
\hline $\log \mathcal{L}$ & -3642.944 & & \\
Number of parameters & 9 & & \\
Number of observations & 119156 & & \\
\hline
\end{tabular}

Table 8: Estimation results - Delft case study

The positive sign of the parameter $a_{\alpha}$ shows that $\alpha_{k}$, that is the likelihood of low speeds, increases with density. Similarly, the positive sign of the parameter $a_{\beta}$ shows that $\beta_{k}$, that is the likelihood of the mode of the speed distribution, also increases with density.

The signs and the estimated values of the parameters $v_{f}, \theta$ and $\gamma$ of the model (15) are in accordance with the trend observed in the data.

The value of the parameter $\lambda$ is higher than the one for the Lausanne case study, which is consistent with the reduced range of speed values in the data. Finally, the value of the parameter $\eta$ is slightly lower than that of Lausanne case study.

\subsection{Comparison with deterministic models}

The performance of the proposed model is compared with the deterministic models proposed in the literature (Table 2), using the same procedure as for the Lausanne case study. The models are compared in Figure 13 and the goodness of fit measures are reported in Table 9 Again, PedProb-vk exhibits the best fit. 


\begin{tabular}{|c|c|c|c|c|c|}
\hline Model & Weidmann (1993) & Tregenza (1976) & Rastogi et al. (2013) & Linear & PedProb-vk \\
\hline$M S E$ & $3.79 e^{-02}$ & $1.93 e^{-62}$ & $3.37 e^{-02}$ & $4.56 e^{-02}$ & $1.69 e^{-02}$ \\
\hline $\bar{R}^{2}$ & $3.58 e^{-01}$ & $6.74 e^{-01}$ & $4.31 e^{-01}$ & $2.29 e^{-01}$ & $7.15 e^{-01}$ \\
\hline
\end{tabular}

Table 9: Goodness of Fit (aggregate level) - Delft case study

\subsection{Kolmogorov-Smirnov validation}

The agreement between the model predictions and the observations from the estimation dataset is illustrated in Figure 14 and Figure 15. The Kolmogorov-Smirnov distances (27) between the model cdf and the empirical cdf are illustrated in the Figure 16a.

The agreement between the model predictions with data appears to be less satisfactory for lower density levels, as we have fewer data with low speed at low density levels. This is an artefact of the experimental nature of the data (see discussion in Section 5.3). The quality of the fit for higher density levels (which is of greater interest for applications anyway) is satisfactory. Figure 16a shows that the smallest Kolmogorov-Smirnov distances correspond to the density levels which are characterized by the largest number of observations (Table 7).

The $p$-values are estimated using the procedure described in Section 6.2 and shown in Figure 16b Again, there is no evidence in the data to reject PedProb-vk at significance level 0.05 for most of the density levels. The $p$-values less than 0.05 are observed for lower density levels, up to $0.3 \mathrm{ped} / \mathrm{m}^{2}$, and for density levels greater than $3.5 \mathrm{ped} / \mathrm{m}^{2}$. In the former case low $p$-values are caused by the experimental nature of the data (as discussed above), while in the latter a low number of observations $(0.02 \%$ of the data) is insufficient to reach any conclusion.

\subsection{Specification test}

We test the robustness of the model specification by performing the validation using $80 \%$ of the data for estimation and the remaining $20 \%$ for validation (see Section 6.3). The Kolmogorov-Smirnov statistics for different density levels from 100 simulation runs and corresponding $p$-values are shown using box plot representation in Figure $17 \mathrm{a}$ and Figure $17 \mathrm{~b}$ respectively. The above results validate the model also for the Delft case study.

\section{Conclusion and future work}

In this paper a novel speed-density relationship for pedestrian traffic is proposed. Different from the deterministic approaches in the literature, it is a probabilistic model designed to account for the heterogeneity of speed at a given density level, as observed in the data.

Various tests on two different case studies validate the specification of the model. Moreover, the model is shown to outperform traditional deterministic models at the aggregate level as well. 
The presented work has both theoretical and practical implications. It can be combined with a conservation principle in dynamic continuum and discrete models (Hughes, 2002 Hänseler et al. 2014), leading to probabilistic conservation laws for the representation of the pedestrian dynamics. This would allow the detailed analysis of the effects of heterogeneity on pedestrian flows. The suggested model may be utilized as such by practitioners for the evaluation and optimization of the level of service of pedestrian facilities. Contrasted with existing approaches, it yields a more realistic representation of the empirically observed phenomena.

Nonetheless, some aspects require further investigation.

First, the described framework as such is insufficient to explain the multi-directional nature of pedestrian flows. As further steps we will explore the possibility of addressing this issue by adapting the existing definitions of pedestrian traffic characteristics through a stream-based approach and a data-driven spatio-temporal discretization framework (Nikolić and Bierlaire, 2014).

Second, the structure of the unobservables could be further investigated (e.g. explicit definition of individual random effects - which layers do they affect and how, existence of autocorrelation patterns in the unobservables).

Third, the speed dynamics (e.g. state dependence) may be considered as well. In this case the data generating process would be different from the one assumed in this paper, and all the above statements and estimation method would need to be reconsidered. In the presence of lagged speeds and random individual effects there is correlation between unobservables and the lagged dependent variable. The current quasi-maximum likelihood estimator would lead to inconsistent estimates, while the full information maximum likelihood estimator would involve more simulation-based inference and working on the initial value problem. Therefore conditional maximum likelihood estimation using a correction as proposed by Wooldridge (2010) would be a good starting methodological point to extent the model.

Finally, we plan to combine this data driven approach with a behavioral approach, where the heterogeneity of speeds is explicitly explained, using variables such as trip purpose, walking in groups, train timetables, etc.

\section{Appendix}

The integral (17) can be simplified in some circumstances and can be solved in a closed form. Consider the following probability density function of the speed that is defined on the interval $\left[v_{e}(k)-\omega(k), v_{e}(k)+\omega(k)\right]$

$$
f_{V}\left(\xi \mid v_{e}(k), \omega(k)\right)=\left\{\begin{array}{lr}
\frac{\xi-v_{e}(k)+\omega(k)}{\omega(k){ }^{2}}, & v_{e}(k)-\omega(k) \leq \xi \leq v_{e}(k) \\
\frac{v_{e}(k)+\omega(k)-\xi}{\omega(k)^{2}}, & v_{e}(k) \leq \xi \leq v_{e}(k)+\omega(k) \\
0, & \xi<v_{e}(k)-\omega(k) \text { or } \xi>v_{e}(k)+\omega(k) .
\end{array}\right.
$$

If the symmetric triangular distribution (22) is the assumed mixing distribution, the 
resulting probabilistic speed-density relationship is given as

$$
f_{\text {PedProb-vk }}\left(\xi \mid k ; \bar{v}_{e}(k), \omega(k), \sigma(k)\right)=\left\{\begin{array}{rr}
\frac{\omega(k)+\xi-\bar{v}_{e}(k)}{\omega^{2}(k)}, & \xi \leq \bar{v}_{e}(k)-\sigma(k) \\
\frac{\omega(k)-\frac{\sigma(k)}{3}}{\omega(k)}-\frac{\left(\xi-\bar{v}_{e}(k)\right)^{2}}{\sigma(k) \omega(k)}-\frac{\left(\xi-\bar{v}_{e}(k)\right)^{3}}{3 \sigma(k)^{2} \omega(k)^{2}}, & \bar{v}_{e}(k)-\sigma(k)<\xi \leq \bar{v}_{e}(k) \\
\frac{\omega(k)-\frac{\sigma(k)}{3}}{\omega(k) \bar{v}^{2}}-\frac{\left(\xi-\bar{v}_{e}(k)\right)^{2}}{\sigma(k) \omega(k))^{2}}+\frac{\left(\xi-\bar{v}_{e}(k)\right)^{3}}{\left.3 \sigma(k)^{2} \omega(k)\right)^{2},}, & \bar{v}_{e}(k)<\xi \leq \bar{v}_{e}(k)+\sigma(k) \\
\frac{\omega(k)-\xi+\bar{e}_{e}(k)}{\omega^{2}(k)}, & \xi>\bar{v}_{e}(k)+\sigma(k) .
\end{array}\right.
$$

Similarly, if we assume the uniform mixing distribution defined on the interval $\left[\bar{v}_{e}(k)-\right.$ $\left.\alpha(k), \bar{v}_{e}(k)+\alpha(k)\right]$

$$
f_{v_{e}(k)}\left(\zeta ; \bar{v}_{e}(k), \alpha(k)\right)=\left\{\begin{array}{lr}
\frac{1}{2 \alpha(k)}, & \bar{v}_{e}(k)-\alpha(k) \leq \zeta \leq \bar{v}_{e}(k)+\alpha(k) \\
0, & \zeta<\bar{v}_{e}(k)-\alpha(k) \text { or } \zeta>\bar{v}_{e}(k)+\alpha(k),
\end{array}\right.
$$

the resulting probabilistic speed-density relationship is given as

$$
f_{\text {PedProb-vk }}\left(\xi \mid k ; \bar{v}_{e}(k), \omega(k), \alpha(k)\right)=\left\{\begin{array}{lr}
\frac{\xi+\omega(k)-\bar{v}_{e}(k)}{\omega(k)^{2}}, & \xi \leq \bar{v}_{e}(k)-\alpha(k) \\
-\frac{\alpha(k)-2 \omega(k)}{2 \omega(k)}-\frac{\left(\xi-\bar{v}_{e}(k)\right)^{2}}{2 \alpha(k) \omega(k)^{2}}, & \bar{v}_{e}(k)-\alpha(k)<\xi \leq \bar{v}_{e}(k)+\alpha(k) \\
\frac{-\xi+\omega(k)+\bar{v}_{e}(k)}{\omega(k)^{2}}, & \xi>\bar{v}_{e}(k)+\alpha(k) .
\end{array}\right.
$$




\section{Acknowledgements}

This research is supported by the Swiss National Science Foundation Grant 200021141099 "Pedestrian dynamics: flows and behavior". The authors would like to thank SBB-CFF-FFS, who provided us with the dataset collected in the Lausanne train station in the framework of the project "PedFlux: Pedestrian flow modeling in train stations", and Winnie Daamen and Serge Hoogendoorn for the data from controlled experiments performed at the Technical University of Delft in the Netherlands. We are also thankful to our group members Riccardo Scarinci, Shadi Sharif Azadeh and Flurin Hänseler for their invaluable assistance and suggestions. We would like to thank the referees of the paper. Their valuable comments helped in improving the quality of the manuscript. 


\section{References}

Alahi, A., Bierlaire, M., Vandergheynst, P., 2014. Robust real-time pedestrians detection in urban environments with a network of low resolution cameras. Transportation Research Part C: Emerging Technologies 39, 113-128.

Alahi, A., Jacques, L., Boursier, Y., Vandergheynst, P., 2011. Sparsity driven people localization with a heterogeneous network of cameras. Journal of Mathematical Imaging and Vision 41 (1-2), 39-58.

Bauer, D., Brandle, N., Seer, S., Ray, M., Kitazawa, K., 2009. Measurement of pedestrian movements: A comparative study on various existing systems. In: Pedestrian Behavior: Models, Data Collection and Applications. Emerald Group Publishing Limited: Bingley, UK.

Blue, V., Adler, J., 1998. Emergent fundamental pedestrian flows from cellular automata microsimulation. Transportation Research Record: Journal of the Transportation Research Board (1644), 29-36

Bowman, B. L., Vecellio, R. L., 1994. Pedestrian walking speeds and conflicts at urban median locations. Transportation research record (1438), 67-73.

Cheah, J. Y., Smith, J. M., 1994. Generalized M/G/c/c state dependent queueing models and pedestrian traffic flows. Queueing Systems 15 (1-4), 365-386.

Cheung, C., Lam, W. H., 1998. Pedestrian route choices between escalator and stairway in mtr stations. Journal of transportation engineering 124 (3), 277-285.

Daamen, W., 2004. Modelling passenger flows in public transport facilities. Ph.D. thesis, Delft University of Technology, Delft.

Daamen, W., Hoogendoorn, S. P., 2003. Controlled experiments to derive walking behaviour. European Journal of Transport and Infrastructure Research 3 (1), 39-59.

Daamen, W., Hoogendoorn, S. P., Bovy, P. H., 2005. First-order pedestrian traffic flow theory. Transportation Research Record: Journal of the Transportation Research Board 1934 (1), 43-52.

Davidson, R., MacKinnon, J. G., 2004. Econometric theory and methods. Vol. 5. Oxford University Press New York.

Davison, A. C., Hinkley, D. V., 1997. Bootstrap methods and their application. Vol. 1. Cambridge university press.

DiNenno, P. J., 2002. SFPE handbook of fire protection engineering. National Fire Protection Association, Quincy, Massachusetts.

Drake, J., Schofer, J., May, A., 1967. A statistical analysis of speed-density hypotheses. Highway Research Record 154, 53-87.

Duives, D., Daamen, W., Hoogendoorn, S., 2014. Anticipation behavior upstream of a bottleneck. Transportation Research Procedia 2, 43-50.

Flötteröd, G., Lämmel, G., 2015. Bidirectional pedestrian fundamental diagram. Transportation research part B: methodological 71, 194-212.

Fruin, J. J., 1971. Designing for pedestrians: A level-of-service concept. No. 355. Highway Research Board, Washington, DC, pp. 1-15.

Gourieroux, C., Monfort, A., Trognon, A., 1984. Pseudo maximum likelihood methods: Theory. Econometrica: Journal of the Econometric Society, 681-700.

Greenshields, B., Bibbins, J., Channing, W., Miller, H., 1935. A study of traffic capacity. In: Proceedings of the Highway Research Board. Vol. 14. Highway Research Board, Washington, DC.

Hair, J. F., Black, W. C., Babin, B. J., Anderson, R. E., 2009. Multivariate data analysis.

Hall, P., Horowitz, J. L., Jing, B.-Y., 1995. On blocking rules for the bootstrap with dependent data. Biometrika 82 (3), 561-574.

Hänseler, F., Bierlaire, M., Farooq, B., Mühlematter, T., 2014. A macroscopic loading model for timevarying pedestrian flows in public walking areas. Transportation Research Part B: Methodological 69, $60-80$.

Helbing, D., Molnar, P., 1995. Social force model for pedestrian dynamics. Physical review E 51 (5) 4282.

Hoogendoorn, S. P., van Wageningen-Kessels, F. L., Daamen, W., Duives, D. C., 2014. Continuum modelling of pedestrian flows: From microscopic principles to self-organised macroscopic phenomena. Physica A: Statistical Mechanics and its Applications 416, 684-694.

Hughes, R. L., 2002. A continuum theory for the flow of pedestrians. Transportation Research Part B: Methodological 36 (6), 507-535. 
Jabari, S. E., Zheng, J., Liu, H. X., 2014. A probabilistic stationary speed-density relation based on Newell's simplified car-following model. Transportation Research Part B: Methodological 68, $205-223$.

Kim, T., Zhang, H., 2008. A stochastic wave propagation model. Transportation Research Part B Methodological 42 (7), 619-634.

Lam, W. H., Morrall, J. F., Ho, H., 1995. Pedestrian flow characteristics in Hong Kong. Transportation Research Record (1487), 56-62.

Liddle, J., Seyfried, A., Steffen, B., Klingsch, W., Rupprecht, T., Winkens, A., Boltes, M., 2011. Microscopic insights into pedestrian motion through a bottleneck, resolving spatial and temporal variations. In: arXiv:1105.1532.

Massey, F. J., 1951. The Kolmogorov-Smirnov test for goodness of fit. Journal of the American statistical Association 46 (253), 68-78.

Navin, F., Wheeler, R., 1969. Pedestrian flow characteristics. Traffic Engineering, Inst Traffic Engr 39.

Newell, G. F., 1961. Nonlinear effects in the dynamics of car following. Operations Research 9 (2) 209-229.

Nikolić, M., Bierlaire, M., 2014. Pedestrian-oriented flow characterization. Transportation Research Procedia 2, 359-366

Nikolić, M., Bierlaire, M., Farooq, B., 2014. Probabilistic speed-density relationship for pedestrians based on data driven space and time representation. In: Proceedings of the Swiss Transportation Research Conference. Ascona, Switzerland.

Okabe, A., Boots, B., Sugihara, K., Chiu, S. N., 2000. Spatial tessellations: concepts and applications of Voronoi diagrams, Second Edition. Wiley, New York.

Older, S., 1968. Movement of pedestrians on footways in shopping streets. Traffic engineering and control $10,160-163$.

Openshaw, S., 1983. The modifiable areal unit problem. In: Concepts and Techniques in Modern Geography. No. 38. Geobooks, Norwich, England.

Rastogi, R., Ilango, T., Chandra, S., 2013. Pedestrian flow characteristics for different pedestrian facilities and situations. European Transport 53.

Ross, S., 2013a. Chapter 11 - Statistical Validation Techniques. In: Simulation (Fifth Edition). Academic Press, pp. $247-270$.

Ross, S., 2013b. Chapter 5 - Generating Continuous Random Variables. In: Simulation (Fifth Edition) Academic Press, pp. $69-96$.

Seyfried, A., Boltes, M., Kähler, J., Klingsch, W., Portz, A., Rupprecht, T., Schadschneider, A., Steffen B., Winkens, A., 2010. Enhanced empirical data for the fundamental diagram and the flow through bottlenecks. In: Pedestrian and Evacuation Dynamics 2008. Springer.

Steffen, B., Seyfried, A., 2010. Methods for measuring pedestrian density, flow, speed and direction with minimal scatter. Physica A: Statistical mechanics and its applications 389 (9), 1902-1910.

Tanaboriboon, Y., Hwa, S. S., Chor, C. H., 1986. Pedestrian characteristics study in singapore. Journal of Transportation Engineering 112 (3), 229-235.

Tregenza, P., 1976. The design of interior circulation. Van Nostrand Reinhold, New York.

Treiber, M., Helbing, D., 2003. Memory effects in microscopic traffic models and wide scattering in flow-density data. Physical Review E 68 (4), 046119

Trognon, A., 1987. Les méthodes du pseudo-maximum de vraisemblance. Annales d'Économie et de Statistique, 117-134.

Underwood, R. T., 1961. Speed, volume, and density relationships: Quality and theory of traffic flow. In: Yale Bureau of Highway Traffic,New Haven.

Wang, H., Li, J., Chen, Q.-Y., Ni, D., 2009. Speed-density relationship: From deterministic to stochastic In: Transportation Research Board 88th Annual Meeting, Washington, DC.

Wasserman, L., 2000. Bayesian model selection and model averaging. Journal of mathematical psychology 44 (1), 92-107

Weidmann, U., 1993. Transporttechnik der fussgänger. Tech. Rep. Schriftenreihe des IVT Nr. 90, Institut für Verkehrsplanung, Transporttechnik,Strassen- und Eisenbahnbau, ETH Zürich, (In German).

Wooldridge, J. M., 2010. Econometric analysis of cross section and panel data. MIT press.

Zhang, J., 2012. Pedestrian fundamental diagrams: Comparative analysis of experiments in different geometries. $\mathrm{Ph} . \mathrm{D}$. thesis, Forschungszentrum Jülich. 


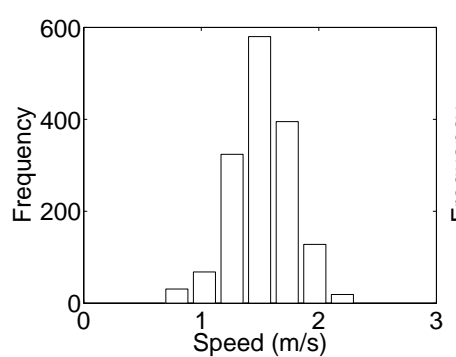

(a) $\mathrm{k}<0.1 \mathrm{ped} / \mathrm{m}^{2}$

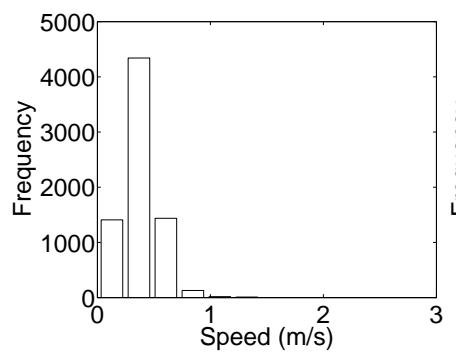

(d) $\mathrm{k}=2 \mathrm{ped} / \mathrm{m}^{2}$

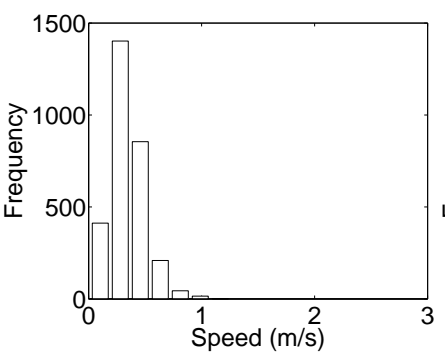

(g) $\mathrm{k}=2.8 \mathrm{ped} / \mathrm{m}^{2}$

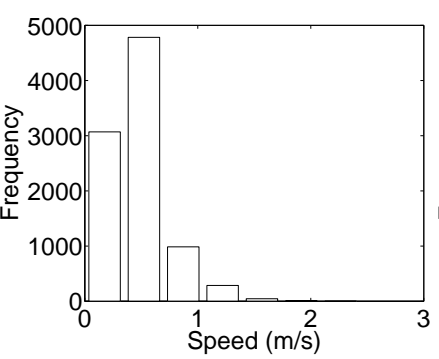

(b) $\mathrm{k}=1 \mathrm{ped} / \mathrm{m}^{2}$

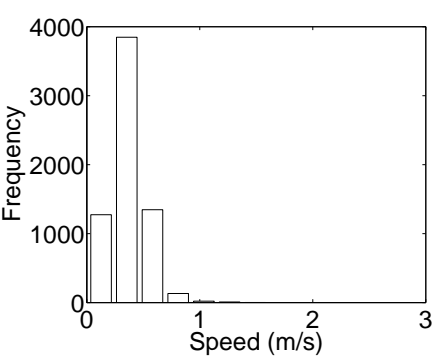

(e) $\mathrm{k}=2.2 \mathrm{ped} / \mathrm{m}^{2}$

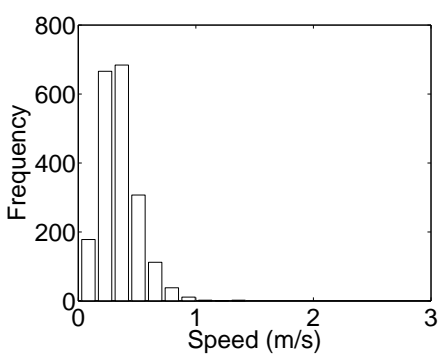

(h) $\mathrm{k}=3 \mathrm{ped} / \mathrm{m}^{2}$

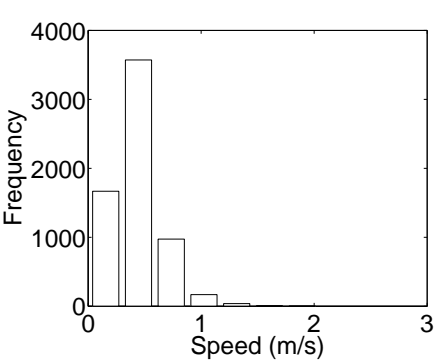

(c) $\mathrm{k}=1.5 \mathrm{ped} / \mathrm{m}^{2}$

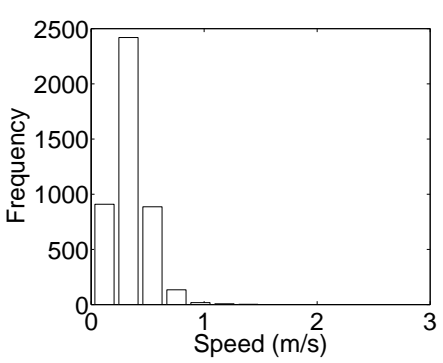

(f) $\mathrm{k}=2.6 \mathrm{ped} / \mathrm{m}^{2}$

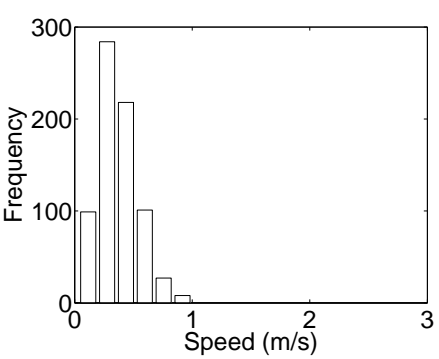

(i) $\mathrm{k}=3.5 \mathrm{ped} / \mathrm{m}^{2}$

Figure 7: Speed distributions for different density levels - Delft case study 


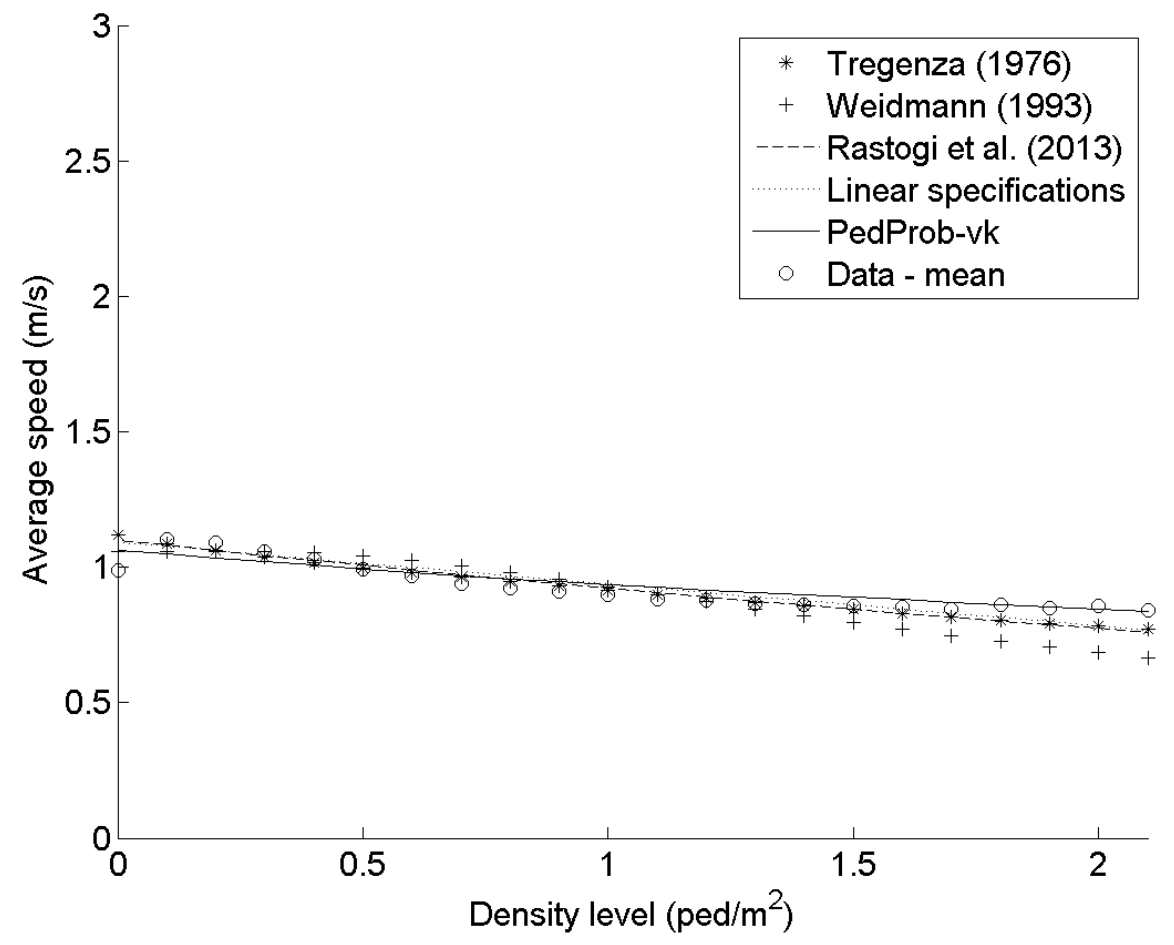

Figure 8: Comparison between deterministic models predictions and the aggregated probabilistic model predictions - Lausanne case study 

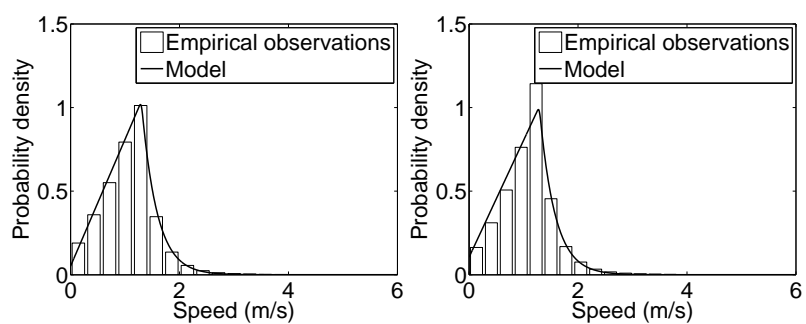

(a) $\mathrm{k}<0.1 \mathrm{ped} / \mathrm{m}^{2}$ (156,578 observations)

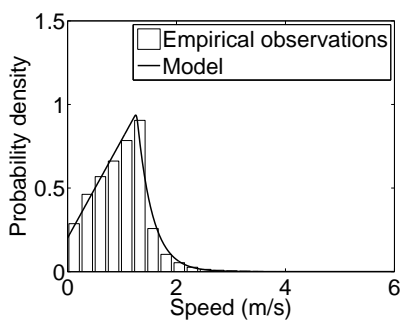

(d) $\mathrm{k}=0.6 \mathrm{ped} / \mathrm{m}^{2}$ (148,500 observations)

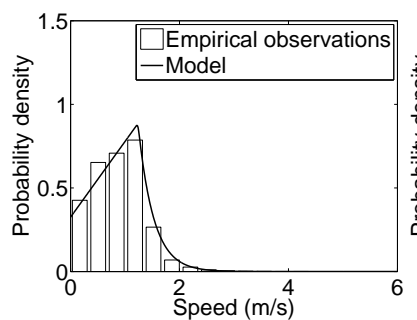
(g) $\mathrm{k}=1.2 \mathrm{ped} / \mathrm{m}^{2}$

(47,727 observations) (b) $\mathrm{k}=0.2 \mathrm{ped} / \mathrm{m}^{2}$ (470,223 observations)

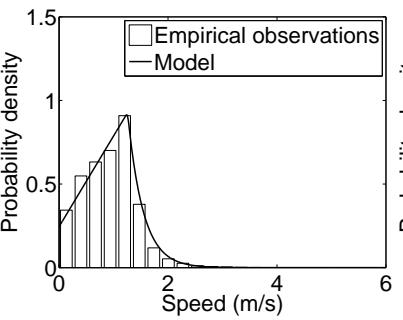

(e) $\mathrm{k}=0.8 \mathrm{ped} / \mathrm{m}^{2}$ (130,962 observations)

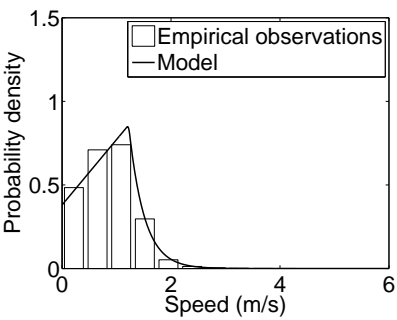

(h) $\mathrm{k}=1.5 \mathrm{ped} / \mathrm{m}^{2}$

(23,828 observations)

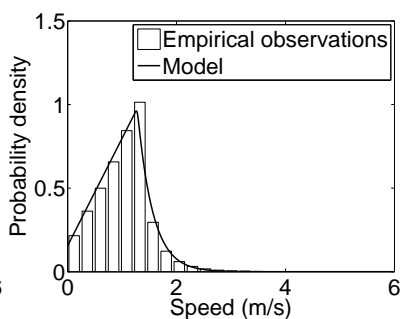

(c) $\mathrm{k}=0.4 \mathrm{ped} / \mathrm{m}^{2}$ (267,644 observations)

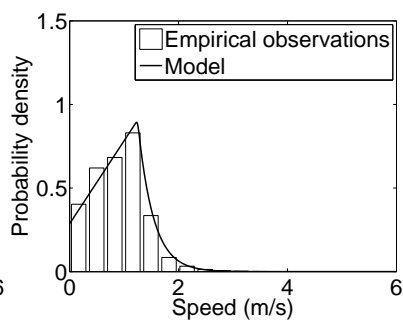

(f) $\mathrm{k}=1 \mathrm{ped} / \mathrm{m}^{2}$ (77,909 observations)

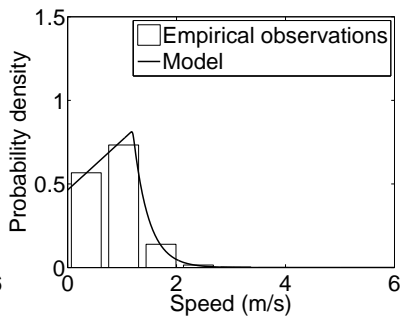

(i) $\mathrm{k}=2 \mathrm{ped} / \mathrm{m}^{2}$

(11,148 observations)

Figure 9: Comparison between model predictions (probability density) and empirical observations Lausanne case study 


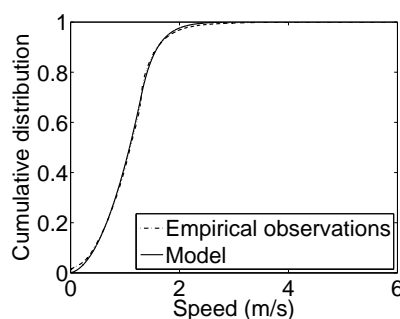

(a) $\mathrm{k}<0.1 \mathrm{ped} / \mathrm{m}^{2}$ (156,578 observations)

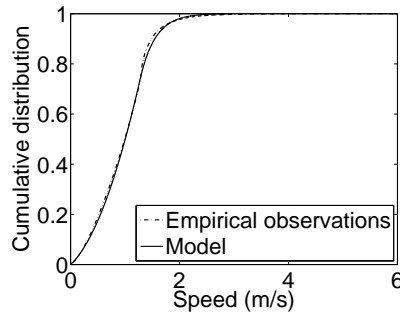

(d) $\mathrm{k}=0.6 \mathrm{ped} / \mathrm{m}^{2}$ (148,500 observations)

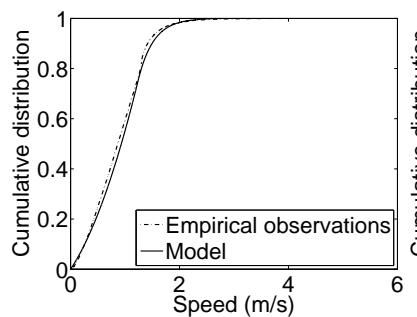

(g) $\mathrm{k}=1.2 \mathrm{ped} / \mathrm{m}^{2}$
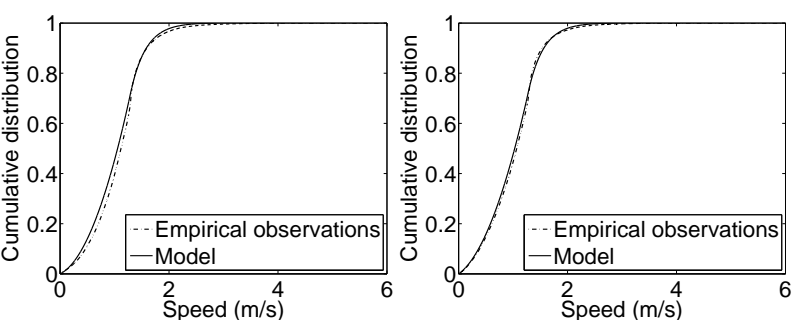

(b) $\mathrm{k}=0.2 \mathrm{ped} / \mathrm{m}^{2}$ (470,223 observations)

(c) $\mathrm{k}=0.4 \mathrm{ped} / \mathrm{m}^{2}$ (267,644 observations)
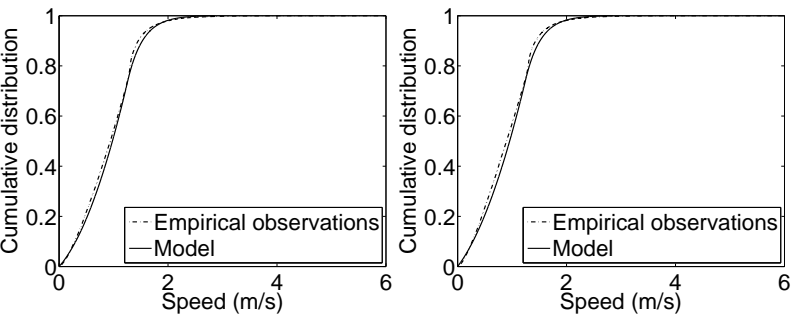

(e) $\mathrm{k}=0.8 \mathrm{ped} / \mathrm{m}^{2}$

(f) $\mathrm{k}=1 \mathrm{ped} / \mathrm{m}^{2}$

(130,962 observations)

(77,909 observations)
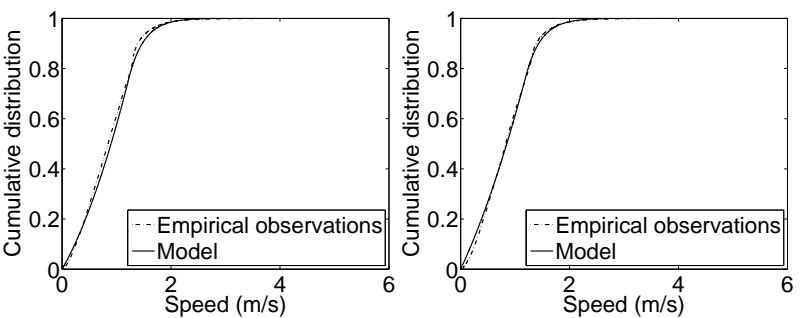

(h) $\mathrm{k}=1.5 \mathrm{ped} / \mathrm{m}^{2}$

(i) $\mathrm{k}=2 \mathrm{ped} / \mathrm{m}^{2}$

(23,828 observations)

Figure 10: Comparison between model predictions (cumulative density) and empirical observations Lausanne case study 


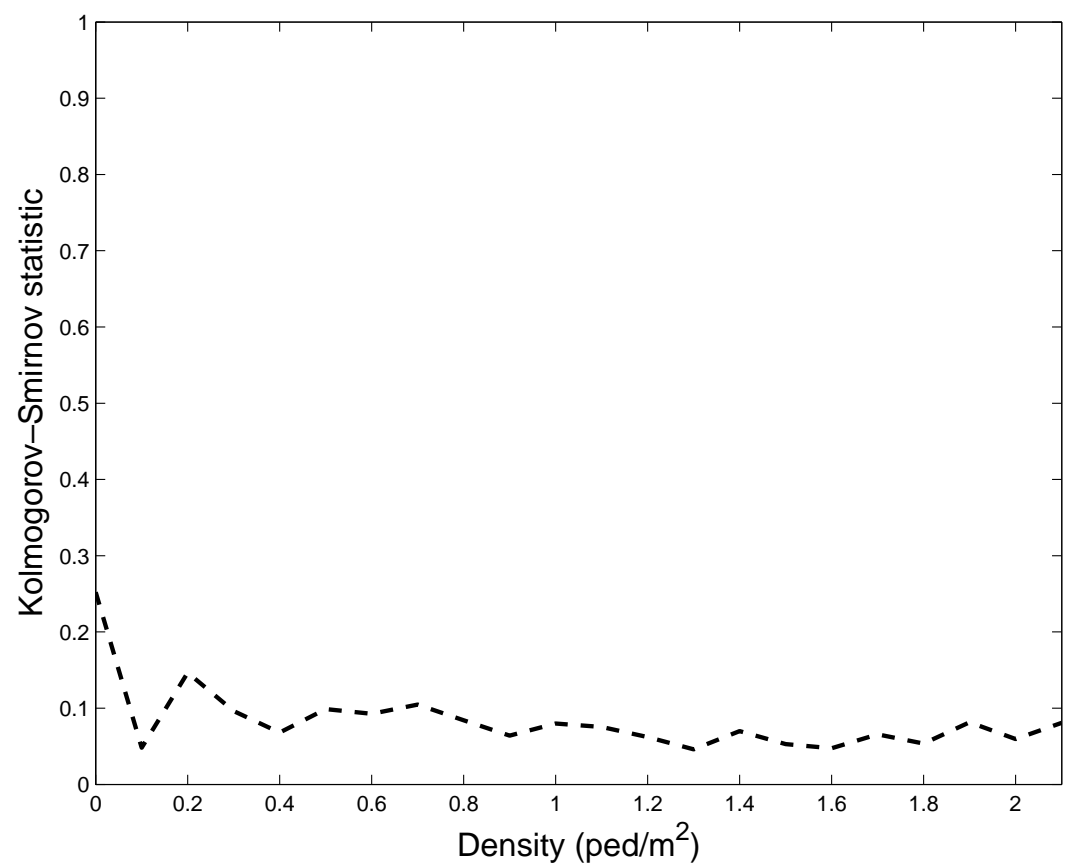

(a) Kolmogorov-Smirnov distance

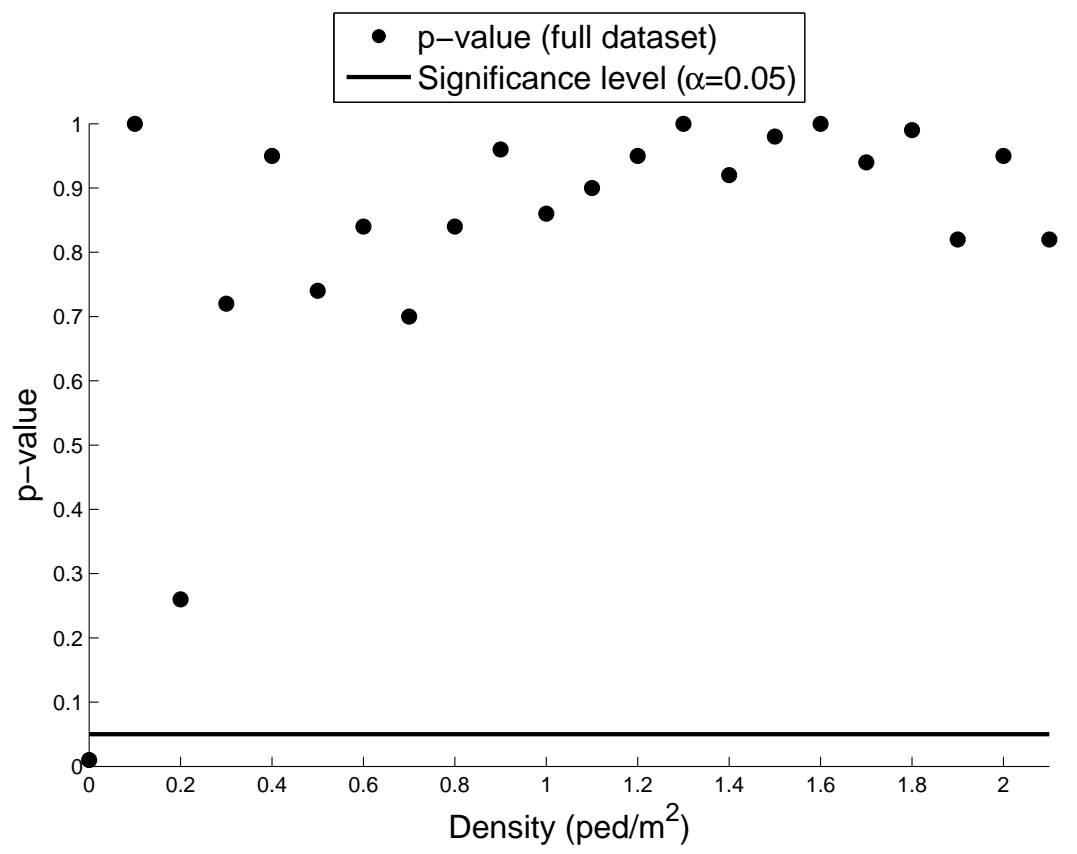

(b) p-value of Kolmogorov-Smirnov statistic

Figure 11: Kolmogorov-Smirnov validation - Lausanne case study 


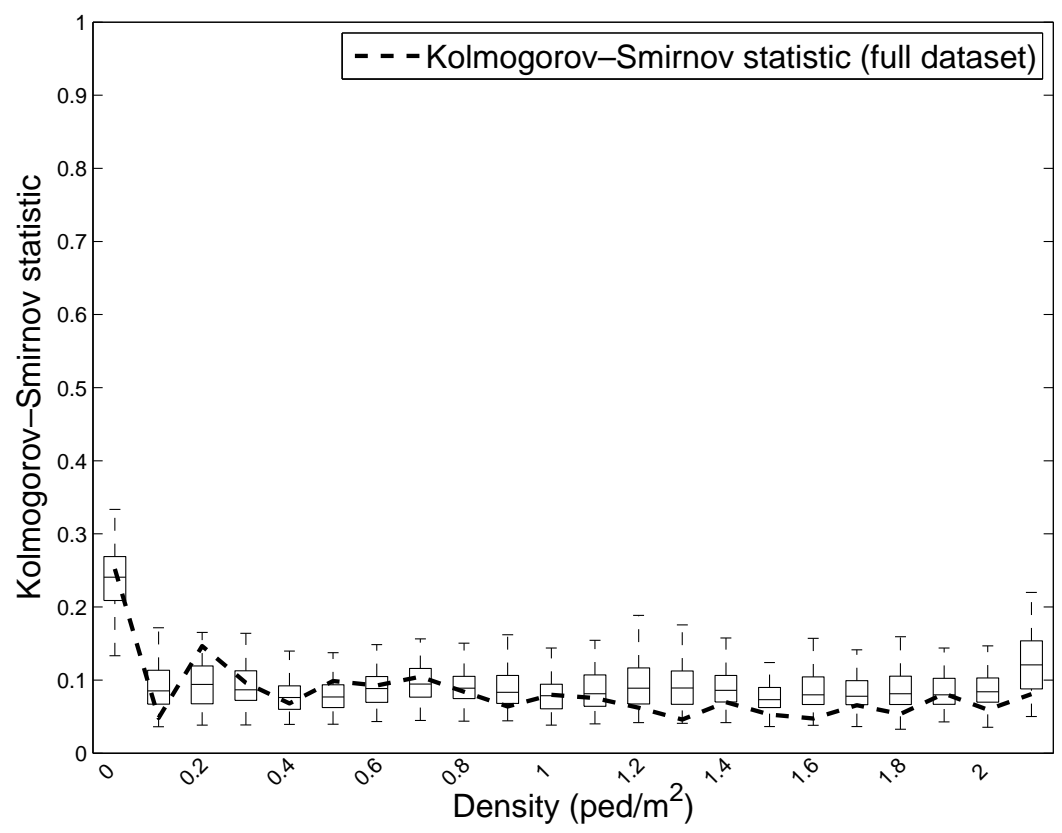

(a) Kolmogorov-Smirnov statistic

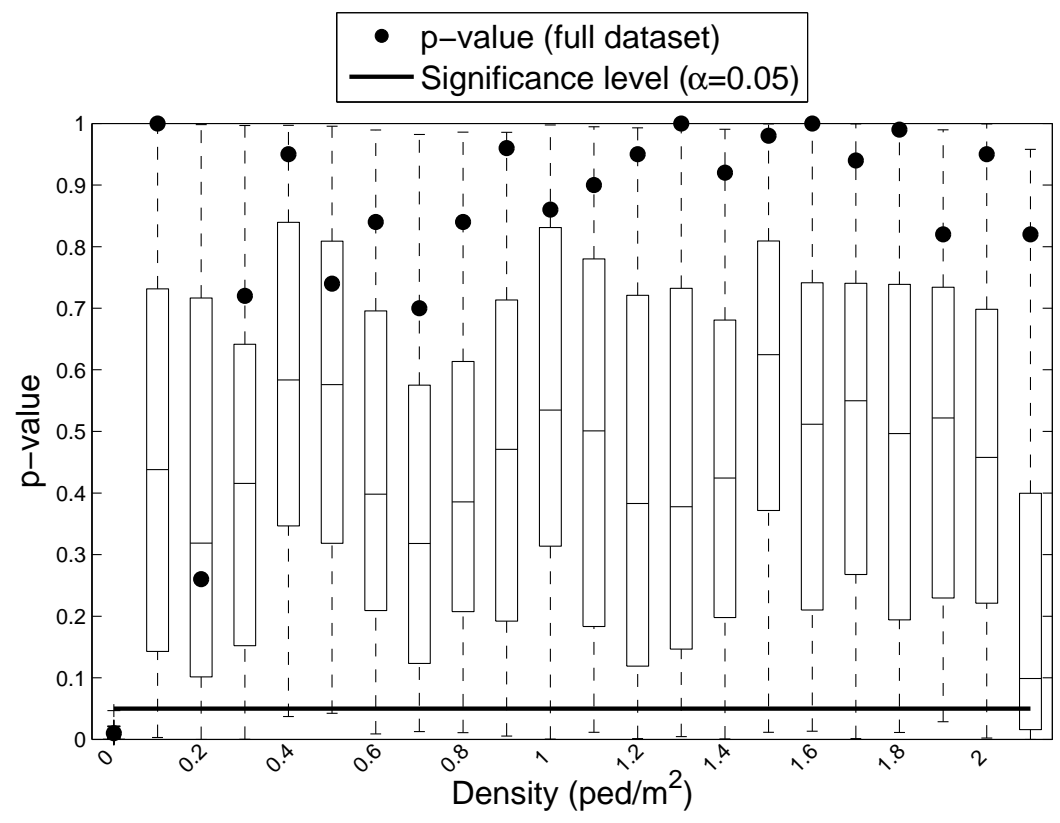

(b) p-value of Kolmogorov-Smirnov statistic

Figure 12: Specification test - Lausanne case study 


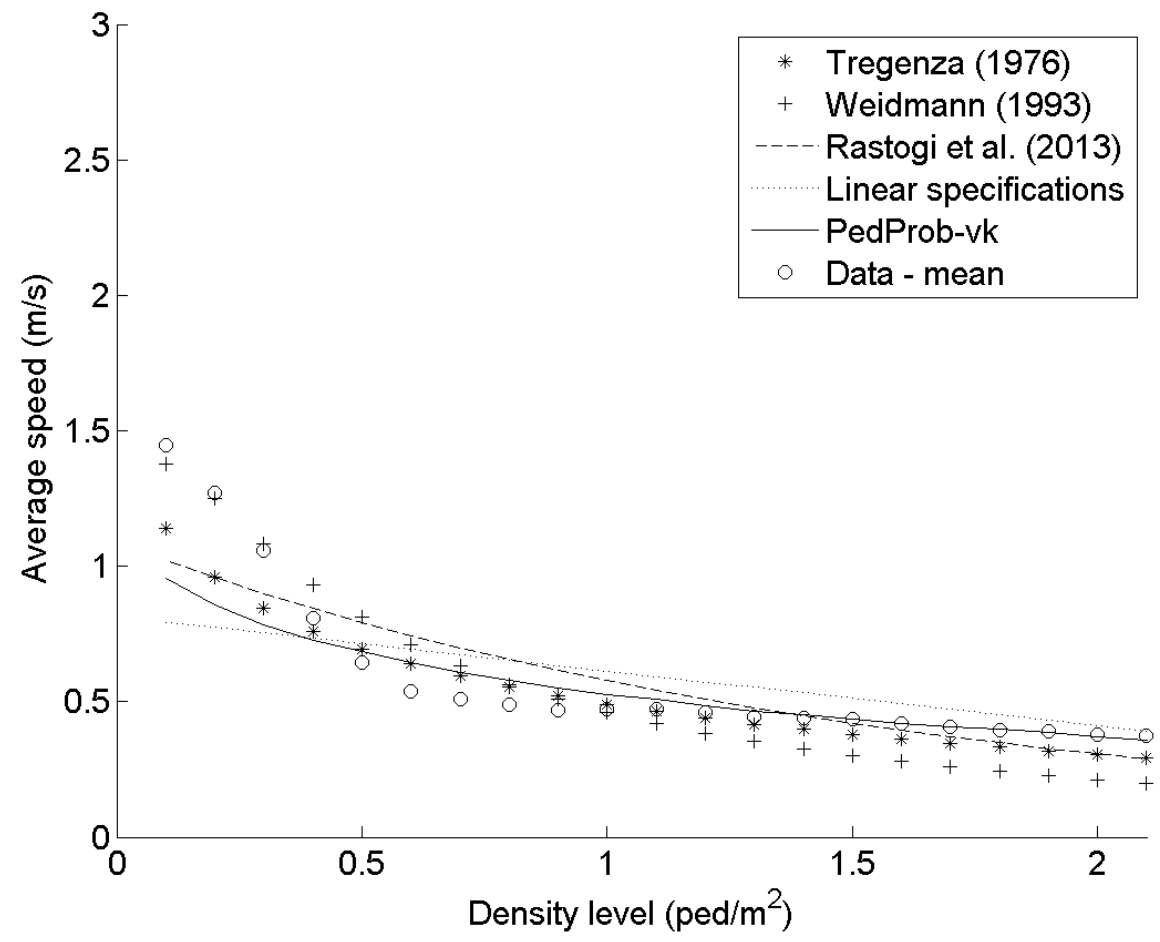

Figure 13: Comparison between deterministic models predictions and the aggregated probabilistic model predictions - Delft case study 

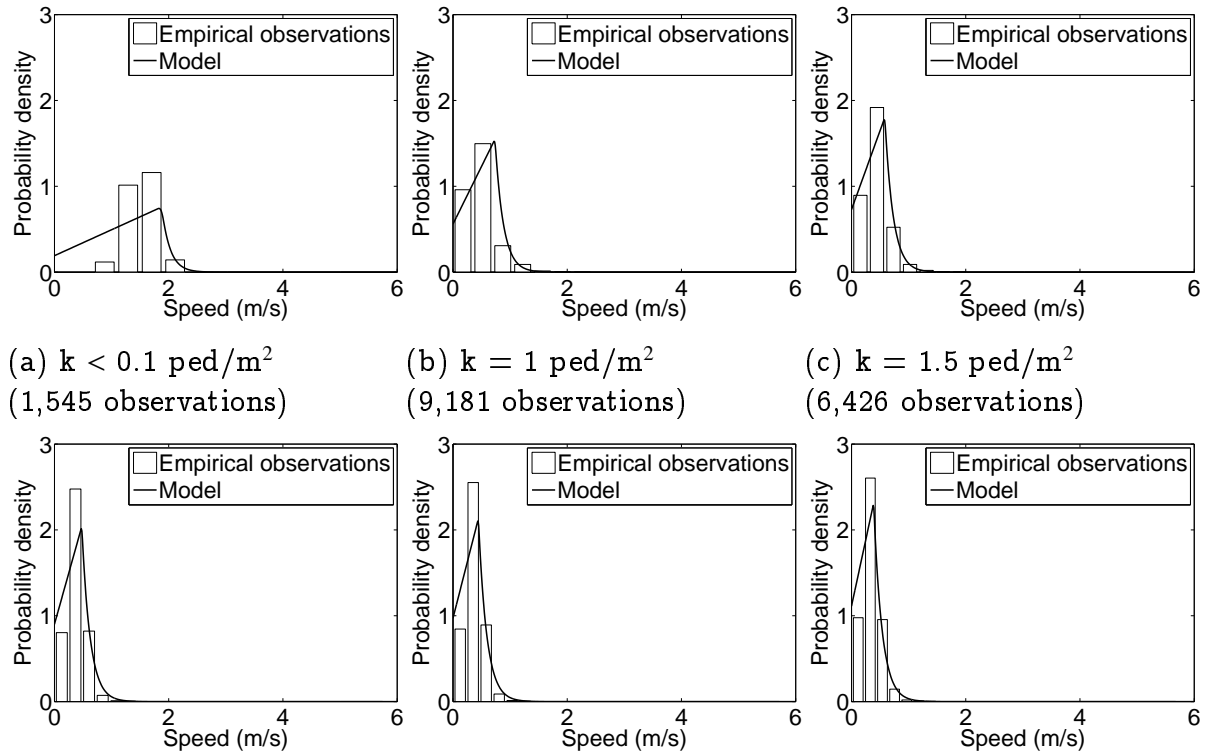

(b) $\mathrm{k}=1 \mathrm{ped} / \mathrm{m}^{2}$

(c) $\mathrm{k}=1.5 \mathrm{ped} / \mathrm{m}^{2}$

(9,181 observations)

(6,426 observations)
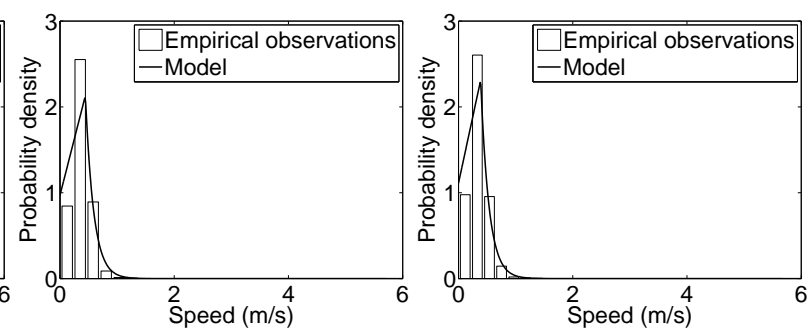

(d) $\mathrm{k}=2 \mathrm{ped} / \mathrm{m}^{2}$

(e) $\mathrm{k}=2.2 \mathrm{ped} / \mathrm{m}^{2}$

(f) $\mathrm{k}=2.6 \mathrm{ped} / \mathrm{m}^{2}$

(7,343 observations)

(6,628 observations)

(4,377 observations)
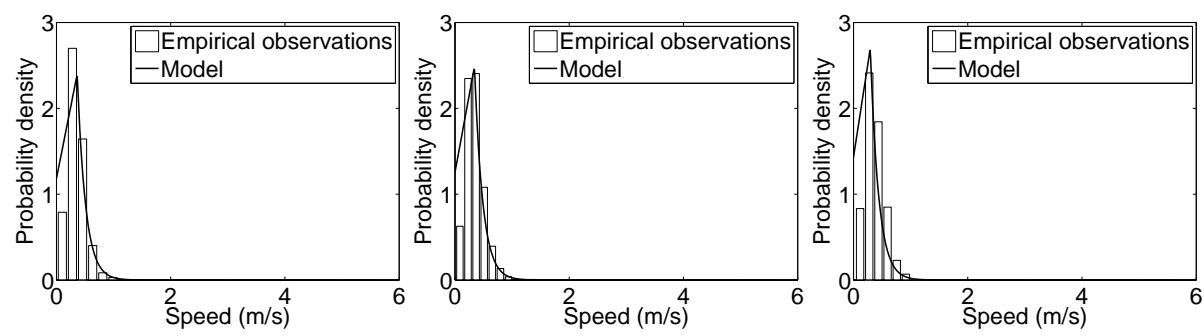
(g) $\mathrm{k}=2.8 \mathrm{ped} / \mathrm{m}^{2}$
(h) $\mathrm{k}=3 \mathrm{ped} / \mathrm{m}^{2}$
(i) $\mathrm{k}=3.5 \mathrm{ped} / \mathrm{m}^{2}$
(2,939 observations)
(2,001 observations)
(737 observations)

Figure 14: Comparison between model predictions (probability density) and empirical observations Delft case study 

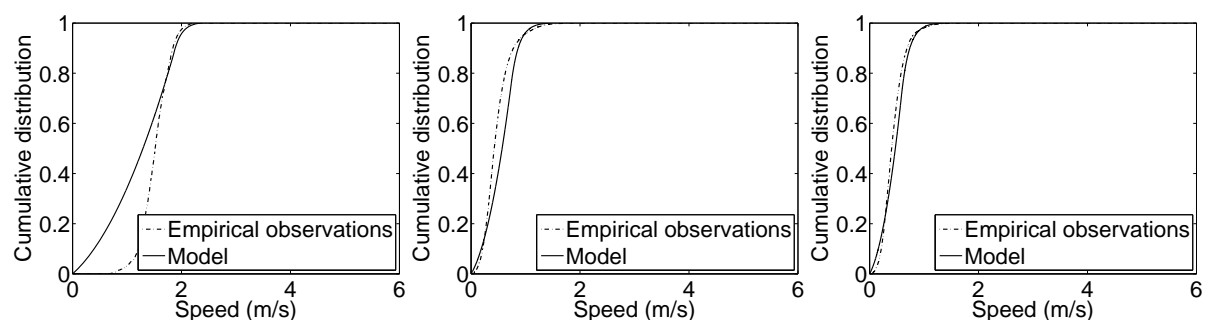

(a) $\mathrm{k}<0.1 \mathrm{ped} / \mathrm{m}^{2}$

(b) $\mathrm{k}=1 \mathrm{ped} / \mathrm{m}^{2}$

(c) $\mathrm{k}=1.5 \mathrm{ped} / \mathrm{m}^{2}$

(1,545 observations)

( 9,181 observations)

$(6,426$ observations $)$
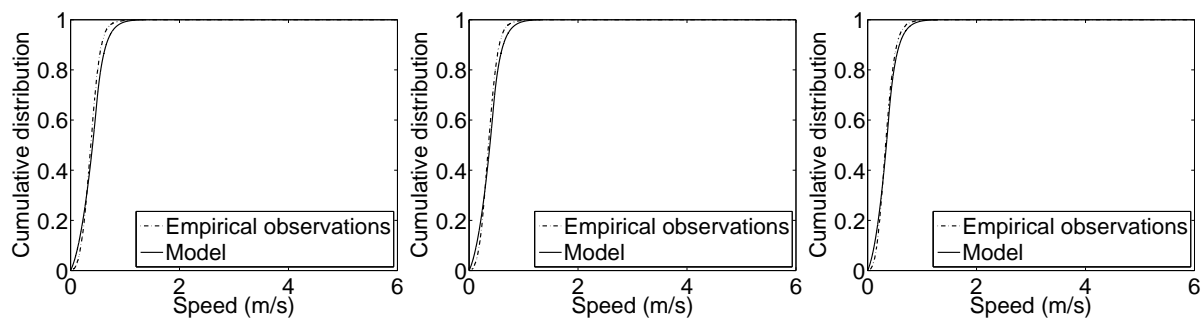

(d) $\mathrm{k}=2 \mathrm{ped} / \mathrm{m}^{2}$

(e) $\mathrm{k}=2.2 \mathrm{ped} / \mathrm{m}^{2}$

(f) $\mathrm{k}=2.6 \mathrm{ped} / \mathrm{m}^{2}$

(7,343 observations)

(6,628 observations)

(4,377 observations)
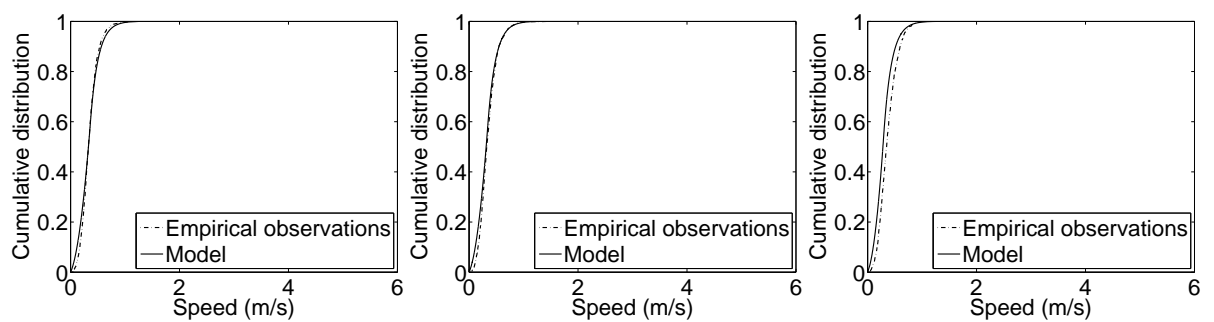
(g) $\mathrm{k}=2.8 \mathrm{ped} / \mathrm{m}^{2}$
(h) $\mathrm{k}=3 \mathrm{ped} / \mathrm{m}^{2}$
(i) $\mathrm{k}=3.5 \mathrm{ped} / \mathrm{m}^{2}$
(2,939 observations)
(2,001 observations)
(737 observations)

Figure 15: Comparison between model predictions (cumulative density) and empirical observations Delft case study 


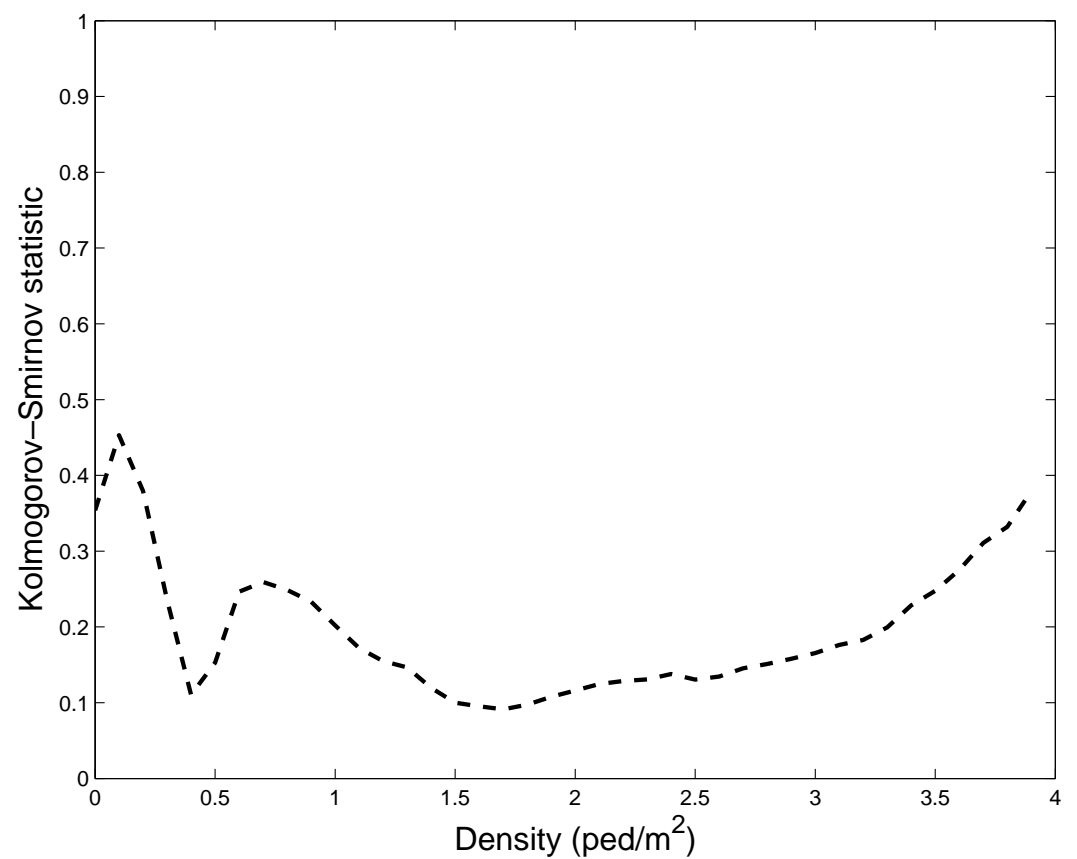

(a) Kolmogorov-Smirnov distance

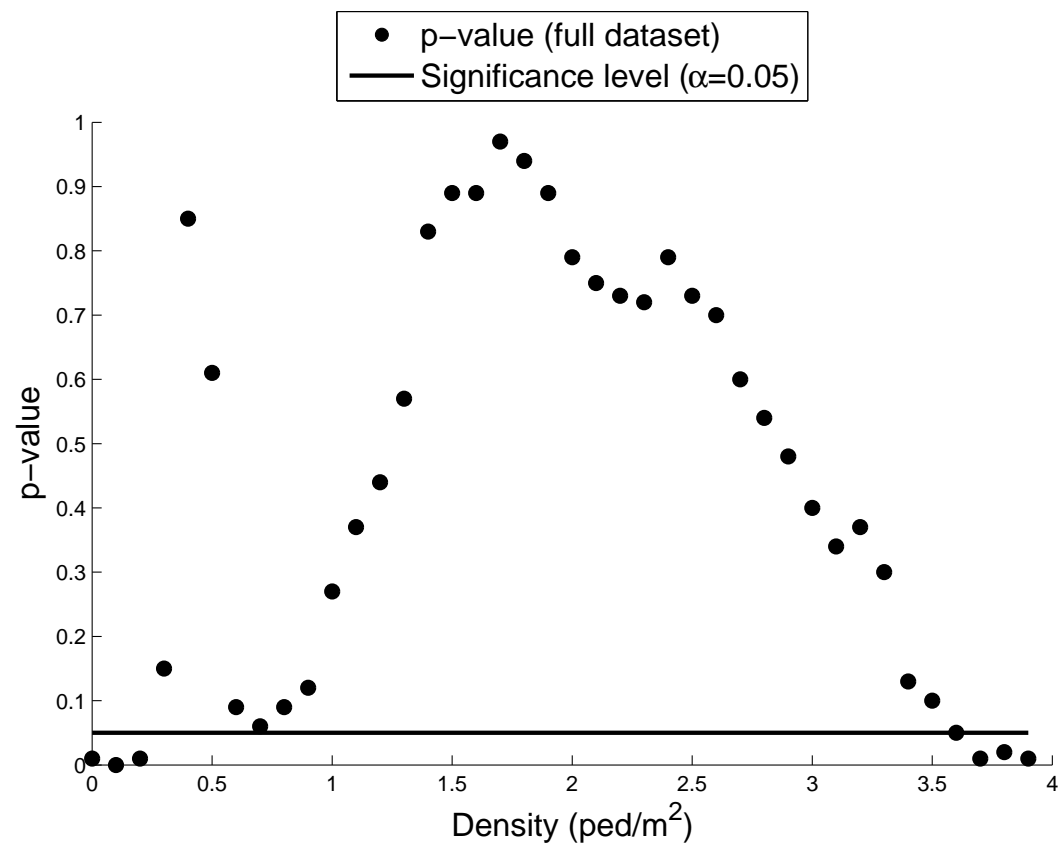

(b) p-value of Kolmogorov-Smirnov statistic

Figure 16: Kolmogorov-Smirnov validation - Delft case study 


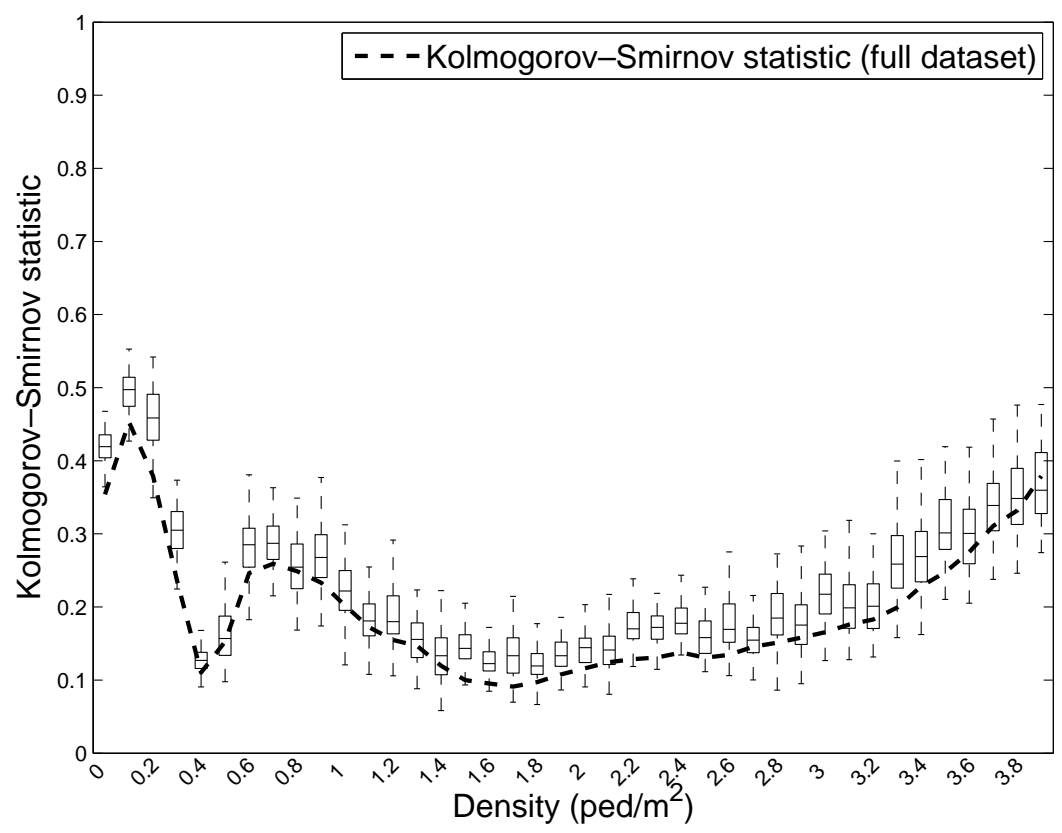

(a) Kolmogorov-Smirnov statistic

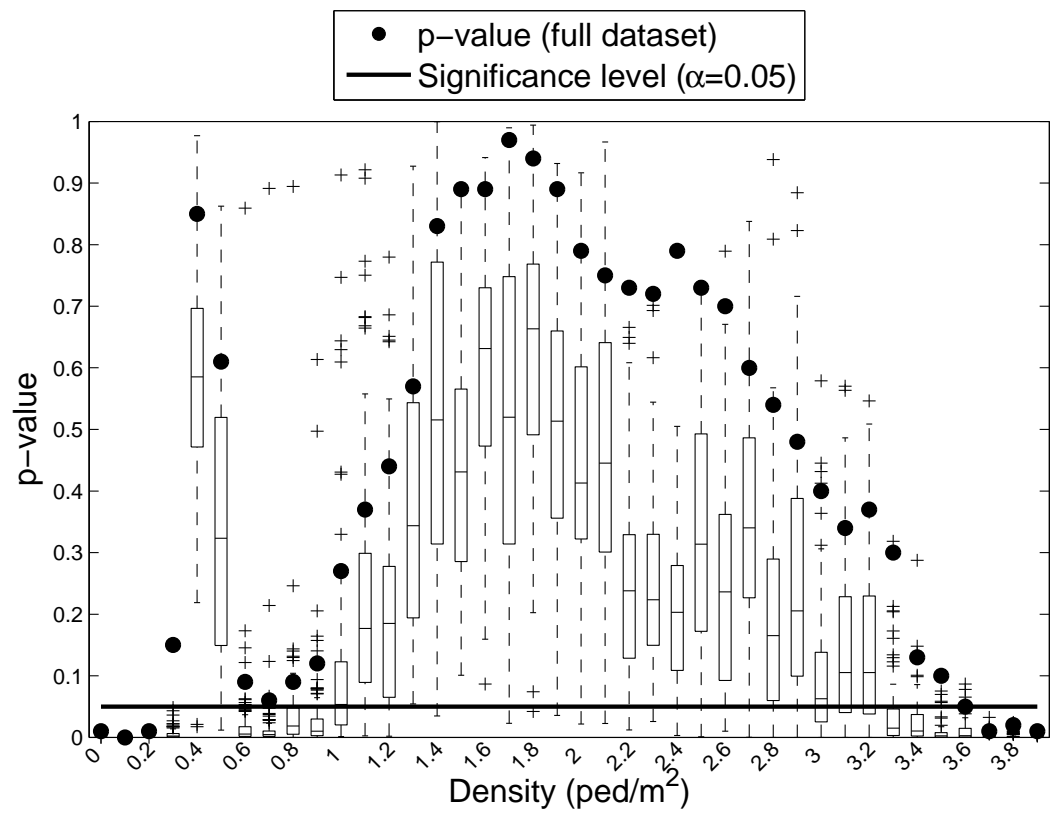

(b) p-value of Kolmogorov-Smirnov statistic

Figure 17: Specification test - Delft case study 